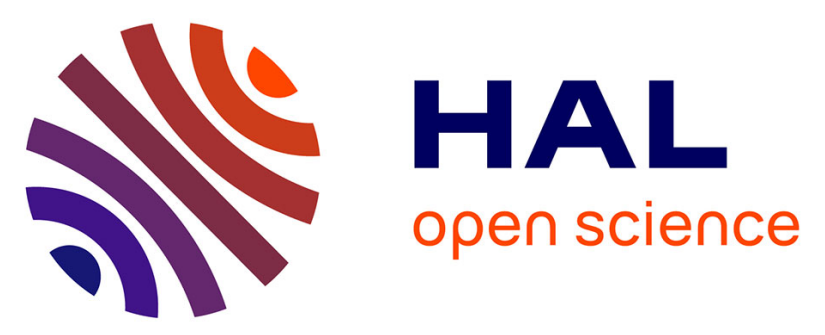

\title{
CRB analysis of planar antenna arrays for optimizing near-field source localization
}

Jean-Pierre Delmas, Mohammed Nabil El Korso, Houcem Gazzah, Marc

Castella

\section{- To cite this version:}

Jean-Pierre Delmas, Mohammed Nabil El Korso, Houcem Gazzah, Marc Castella. CRB analysis of planar antenna arrays for optimizing near-field source localization. Signal Processing, 2016, 127, pp.117 - 134. 10.1016/j.sigpro.2016.02.021 . hal-01355428

\section{HAL Id: hal-01355428 \\ https://hal.science/hal-01355428}

Submitted on 23 Aug 2016

HAL is a multi-disciplinary open access archive for the deposit and dissemination of scientific research documents, whether they are published or not. The documents may come from teaching and research institutions in France or abroad, or from public or private research centers.
L'archive ouverte pluridisciplinaire HAL, est destinée au dépôt et à la diffusion de documents scientifiques de niveau recherche, publiés ou non, émanant des établissements d'enseignement et de recherche français ou étrangers, des laboratoires publics ou privés. 


\title{
CRB analysis of planar antenna arrays for optimizing near-field source localization
}

\author{
Jean Pierre Delmas, Mohammed Nabil El Korso, Houcem Gazzah, and Marc Castella
}

\begin{abstract}
This paper focuses on the Cramér Rao bound (CRB) of the azimuth, elevation and range with planar arrays for narrowband near-field source localization, using the exact expression of the time delay parameter. Specifically, the aim of this paper is twofold. First, we derive explicit non-matrix closed-form expressions of approximations of these three CRBs. Second, we use these expressions to optimize near-field source localization. For deriving these expressions, we introduce conditions on the array geometry that allow us to decouple the azimuth, elevation and range parameters to a certain order in $\lambda / r$ (in which $\lambda$ and $r$ denote the wavelength and the range, respectively). The proposed conditions complement those found for a far-field source that ensure the azimuth and elevation estimations are both exactly decoupled and isotropic. A particular attention is given to the popular array configurations which are the concentric uniform circular-based arrays, cross-based and squarebased centro-symmetric arrays which satisfy these conditions. In order to control directions of arrivals (DOA) ambiguity, we propose a new criterion, which allows us to design non-uniform square [resp., cross]-based centrosymmetric array configurations with improved near-field range estimation capabilities without deteriorating the DOA precisions w.r.t. uniform square [resp., cross]-based arrays. Finally, we specify the accuracy of our proposed approximated CRBs'expressions and isotropy's conditions w.r.t. the range and the number of sensors.
\end{abstract}

\section{Index Terms}

Cramér Rao bound (CRB), near-field source localization, azimuth, elevation, range, planar antenna array, sensor's position optimization.

\section{Manuscript accepted to Signal Processing}

\section{INTRODUCTION}

Sensor placement is known to have a significant impact on the source localization capabilities of the antenna array, and the topic starts to attract an increasing research effort [1]-[5]. Performance analysis based on the

Jean Pierre Delmas and Marc Castella are with Telecom SudParis, CNRS UMR 5157, Université Paris Saclay, Evry, France. E-mail: jean-pierre.delmas@it-sudparis.eu, marc.castella@it-sudparis.eu. Mohammed Nabil El Korso is with Université Paris Ouest Nanterre La Défense, LEME laboratory EA 4416, France. E-mail: m.elkorso@u-paris10.fr. Houcem Gazzah is with the Department of Electrical and Computer Engineering, University of Sharjah, 27272, UAE. E-mail: hgazzah@ sharjah.ac.ae. 
CRB is generally preferred because the latter is, at the same time, algorithm-independent and achievable by a number of popular algorithms. Dependence of the CRB on the array configuration has, first, been studied in relation to DOA estimation of far-field sources [7], [8], assuming a planar wavefront is impinging on each sensor. The more challenging near-field case implies a curvature of the waves and a more complicated time delay model parameterized by the source DOA and range too. In the litterature, one can find a plethora of nearfield performance analysis based on an approximate propagation model applicable to the so-called Fresnel zone [9],[10],[11]. Only lately has the exact time delay formula been used for deriving more accurate closed-form expressions of the CRB. This approach, applied to uniform linear arrays (ULA) [12], arbitrary linear arrays [13] and uniform circular arrays (UCA) [14], is extended for the first time, here, to planar antenna arrays.

The aim of this paper is twofold: First, we tackle the problem of the derivation of explicit non-matrix closedform expressions of approximate $\mathrm{CRB}$ of the azimuth, elevation and range with for narrowband near-field source localization by means of planar arrays. Those derivations are based on the exact expression of the time delay parameter which is very challenging due to the non-linearity of the exact propagation model. Concentrated on the azimuth, elevation and range parameters $(\theta, \phi, r)$, the stochastic and deterministic CRBs, that are proportional (one to the other), are given by the inverse of a Fisher-like information matrix, whose terms are nonlinear expressions of $\theta, \phi, r$, and the coordinates of the sensors. To obtain simple and interpretable expressions of the CRBs on $\theta, \phi$ and $r$, we first specify conditions on the coordinates of the sensors that allow us to decouple $\theta$, $\phi$ and $r$ to a certain order in $1 / r$ for near-field sources. Using Taylor expansions, we explicit the expressions of the CRBs for three classes of planar arrays that satisfy these conditions: the concentric uniform circular based-arrays, the square-based and cross-based centro-symmetric arrays. In particular, we study decoupling in relationship with isotropy which is the array ability to exhibit the same accuracy in all azimuth look directions. In the far-field, estimation is decoupled if and only if it is isotropic. In contrast, we prove that, in the array near-field, the condition that ensures decoupling does not assure exact isotropy.

Second, we focus on the class of square and cross-based centro-symmetric arrays and highlight some of their attractive features. In particular, we identify key geometric parameters that control the near-field array performance. Opportunistically, these geometric parameters are used to design non-uniform square and crossbased centro-symmetric arrays that achieve better near-field localization accuracy. More precisely, this design reduces (by as much as 60\%) the CRB of the range parameter, with identical azumuth's and elevation's CRB as their corresponding uniform square and cross-based arrays. Finally, it should be noted that the proposed CRB-minimizing criterion incorporates some geometric constrains to account for the array ambiguity problem.

The paper is organized as follows. Section II specifies the data model, formulates the problem and gives the general expression of the CRB. In Section III, we use Taylor expansions to derive the CRB for planar arrays. We focus on the following three classes which happen to exhibit decoupled estimation of the source parameters: the concentric uniform circular based-arrays, the square-based and cross-based centro-symmetric arrays. An 
analysis of these CRBs is presented in Section IV while paying attention to isotropy and its dependency on the source range and the number of sensors. We also design original non-uniform square and cross-based centro-symmetric arrays with improved near-field angle and range estimation capabilities w.r.t. their uniform counter-parts. The paper is concluded in Section V. Note that the part dedicated to the UCA with a single circle has been partially presented in [14].

\section{DATA MODEL AND GENERAL EXPRESSION OF THE CRB}

\section{A. Data Model}

A planar antenna array is made of $P$ omni-directional sensors $\left(C_{p}\right)_{p=1, \ldots, P}$ placed in the $[O, x, y)$ plane, at coordinates $\left(x_{p}, y_{p}\right)_{p=1, \ldots, P}$. Without loss of generality, we assume the array centroid to be at the origin $O$ of this plane. A source $S$ located in the antenna array near-field has its position characterized by an azimuth angle $\theta \in[0,2 \pi]$, an elevation angle $\phi \in[0, \pi / 2]$ and a range $r$ (grouped in the vector $\boldsymbol{\alpha}=[\theta, \phi, r]^{T}$ ), as illustrated in Fig.1 for the concentric uniform circular based-arrays. The source is radiating a narrow-band signal, with wavelength $\lambda$, in the presence of an additive noise with complex envelope $\mathbf{n}_{k}$. The complex envelope $\mathbf{x}_{k}$ of the signal collected by this array of sensors is modeled as

$$
\mathbf{x}_{k}=s_{k} \mathbf{a}(\boldsymbol{\alpha})+\mathbf{n}_{k}, \quad k=1, \ldots, K,
$$

where $s_{k}$ is the source signal measured at the origin and $\mathbf{a}(\boldsymbol{\alpha})=\left[e^{i \tau_{1}(\boldsymbol{\alpha})}, \ldots, e^{i \tau_{p}(\boldsymbol{\alpha})}, \ldots, e^{i \tau_{P}(\boldsymbol{\alpha})}\right]^{T}$ is the socalled steering vector, where $\tau_{p}(\boldsymbol{\alpha})$ is defined as $\tau_{p}(\boldsymbol{\alpha})=2 \pi\left(S O-S C_{p}\right) / \lambda$ with $S O=r$ and $\left[S C_{p}\right]^{2}=$ $\left(x_{p}-r \sin \phi \cos \theta\right)^{2}+\left(y_{p}-r \sin \phi \sin \theta\right)^{2}+r^{2} \cos ^{2} \phi$ (see Fig.1 dedicated to the concentric uniform array) can be rewritten as

$$
\tau_{p}(\boldsymbol{\alpha})=2 \pi \frac{r}{\lambda}\left(1-\sqrt{\beta_{p}}\right)
$$

with

$$
\beta_{p} \stackrel{\text { def }}{=} 1-2 \sin \phi\left(\frac{x_{p}}{r} \cos \theta+\frac{y_{p}}{r} \sin \theta\right)+\frac{x_{p}^{2}+y_{p}^{2}}{r^{2}}
$$

Based on $K$ snapshots $\left(\mathbf{x}_{k}\right)_{k=1, \ldots, K}$, estimates of $(\theta, \phi, r)$ are obtained using a variety of algorithms, among which a few are capable of achieving asymptotically the stochastic CRB [15] that we adopt as our performance measure of the array accuracy.

\section{B. General expression of the $C R B$}

Expressions of the CRB are available under the usual statistical properties about $s_{k}$ and $\mathbf{n}_{k}$ : (i) $s_{k}$ and $\mathbf{n}_{k}$ are independent, (ii) $\left(\mathbf{n}_{k}\right)_{k=1, \ldots, K}$ are independent, zero-mean circular Gaussian distributed with covariance $\sigma_{n}^{2} \mathbf{I}_{P}$, (iii) $\left(s_{k}\right)_{k=1, \ldots, K}$ are assumed to be either deterministic unknown parameters (the so-called conditional or deterministic model), or independent zero-mean circular Gaussian distributed with variance $\sigma_{s}^{2}$ (the so-called 
unconditional or stochastic model). The associated deterministic and stochastic CRBs (denoted by $\operatorname{CRB}_{\text {det }}(\boldsymbol{\alpha})$ and $\left.\mathrm{CRB}_{\text {sto }}(\boldsymbol{\alpha})\right)$ are, in fact, proportional one to the other [13] following

$$
\mathrm{CRB}_{\mathrm{sto}}(\boldsymbol{\alpha})=\left(1+\frac{\sigma_{n}^{2}}{\|\mathbf{a}(\boldsymbol{\alpha})\|^{2} \sigma_{s}^{2}}\right) \mathrm{CRB}_{\mathrm{det}}(\boldsymbol{\alpha})
$$

where $\sigma_{s}^{2}$ is to be redefined as $\frac{1}{K} \sum_{k=1}^{k}\left|s_{k}\right|^{2}$ in the deterministic model. We define $\mathbf{F}(\boldsymbol{\alpha}) \stackrel{\text { def }}{=}\left[\operatorname{CRB}_{\text {sto }}(\boldsymbol{\alpha})\right]^{-1}$ proved to be equal to

$$
\mathbf{F}(\boldsymbol{\alpha})=c_{\sigma} \operatorname{Re}\left[\|\mathbf{a}(\boldsymbol{\alpha})\|^{2} \mathbf{D}^{H}(\boldsymbol{\alpha}) \mathbf{D}(\boldsymbol{\alpha})-\mathbf{D}^{H}(\boldsymbol{\alpha}) \mathbf{a}(\boldsymbol{\alpha}) \mathbf{a}^{H}(\boldsymbol{\alpha}) \mathbf{D}(\boldsymbol{\alpha})\right],
$$

with $^{1} \mathbf{D}(\boldsymbol{\alpha}) \stackrel{\text { def }}{=}\left[\frac{\partial \mathbf{a}(\boldsymbol{\alpha})}{\partial \theta}, \frac{\partial \mathbf{a}(\boldsymbol{\alpha})}{\partial \phi}, \frac{\partial \mathbf{a}(\boldsymbol{\alpha})}{\partial r}\right]$ and $c_{\sigma} \stackrel{\text { def }}{=} \frac{2 K \sigma_{s}^{4}}{\sigma_{n}^{2}\left(\sigma_{n}^{2}+P \sigma_{s}^{2}\right)}$, which is independent of the source and sensors positions. Throughout this paper, we only consider the stochastic source model, thanks to (3). After some algebraic manipulations, $\mathbf{F}(\boldsymbol{\alpha})$ is more compactly given (element-wise) by the following expression [13]:

$$
\frac{[\mathbf{F}(\boldsymbol{\alpha})]_{i, j}}{c_{\sigma}}=P \sum_{p=1}^{P} \tau_{p, i}^{\prime} \tau_{p, j}^{\prime}-\left(\sum_{p=1}^{P} \tau_{p, i}^{\prime}\right)\left(\sum_{p=1}^{P} \tau_{p, j}^{\prime}\right)
$$

where $\tau_{p, 1}^{\prime} \stackrel{\text { def }}{=} \frac{\partial \tau_{p}(\boldsymbol{\alpha})}{\partial \theta}, \tau_{p, 2}^{\prime} \stackrel{\text { def }}{=} \frac{\partial \tau_{p}(\boldsymbol{\alpha})}{\partial \phi}$ and $\tau_{p, 3}^{\prime} \stackrel{\text { def }}{=} \frac{\partial \tau_{p}(\boldsymbol{\alpha})}{\partial r}$.

\section{CRB DERIVATION FOR PLANAR ARRAYS}

\section{A. Arbitrary planar arrays}

We perform Taylor expansions of (5) and prove in Appendix VI-A that $\left([\mathbf{F}]_{1,1},[\mathbf{F}]_{1,2},[\mathbf{F}]_{2,2}\right),\left([\mathbf{F}]_{1,3},[\mathbf{F}]_{2,3}\right)$ and $[\mathbf{F}]_{3,3}$ are structured as sums of terms of the form $\frac{r^{2}}{\lambda^{2}}\left[\frac{\sum_{\ell=0}^{k} g_{\ell, k}(\theta, \phi) S_{\ell, k-\ell}}{r^{k}}\right], \frac{r}{\lambda^{2}}\left[\frac{\sum_{\ell=0}^{k} g_{\ell, k}(\theta, \phi) S_{\ell, k-\ell}}{r^{k}}\right]$ and $\frac{1}{\lambda^{2}}\left[\frac{\sum_{\ell=0}^{k} g_{\ell, k}(\theta, \phi) S_{\ell, k-\ell}}{r^{k}}\right]$, respectively, where

$$
S_{i, j} \stackrel{\text { def }}{=} \sum_{p=1}^{P} x_{p}^{i} y_{p}^{j}
$$

are purely geometric parameters and $g_{\ell, k}(\theta, \phi)$ are trigonometric polynomials in $\theta$ and $\phi$. Consequently, the matrix $\mathbf{F}(\boldsymbol{\alpha})$ depends on the array geometry through the terms $S_{i, j}$ only (among which $S_{1,0}=S_{0,1}=0$ ). This contrasts with the far-field case in which $[\mathbf{F}]_{1,1},[\mathbf{F}]_{1,2}$ and $[\mathbf{F}]_{2,2}$ depend only on $S_{1,1}, S_{1,2}$ and $S_{2,2}$ (see e.g., [8]).

Derivation of the CRB on the azimuth, elevation and range alone by inversion of $\mathbf{F}(\boldsymbol{\alpha})$ results into very intricate closed-form expressions, in general. Consequently, we are led to focus on cases where CRB expressions are simple and interpretable. In particular, decoupled estimation, of $\theta, \phi$ and $r$ in $\mathbf{F}(\boldsymbol{\alpha})$, is of primary importance. Unlike the far-field case where $\theta$ and $\phi$ can be decoupled, strict decoupling is not possible in the near-field, and

\footnotetext{
${ }^{1}$ Note this matrix $\mathbf{F}(\boldsymbol{\alpha})$ is not a Fisher information matrix concentrated on $(\theta, \phi, r)$ because the geometric parameter $(\theta, \phi, r)$ is not totally decoupled from the other parameters of the Gaussian parametrization in the deterministic and stochastic modeling.
} 
is achieved only to a certain order of $\epsilon=\frac{\lambda}{r}$. We need, first, to express $\mathbf{F}(\boldsymbol{\alpha})$ as a function of $\epsilon$ by conducting a Taylor expansion with respect to $\epsilon$ by expanding each term of $\mathbf{F}(\boldsymbol{\alpha})$ as a weighted sum of unit-less terms

$$
\frac{S_{l, k-l}}{r^{k}}=\frac{S_{l, k-l}}{\lambda^{k}}\left(\frac{\lambda}{r}\right)^{k}=\left(\frac{\sum_{p=1}^{P} x_{p}^{l} y_{p}^{k-l}}{\lambda^{k}}\right) \epsilon^{k},
$$

in which $\left(x_{p}, y_{p}\right)_{p=1, \ldots, P}$ and $\lambda$ are fixed, but $r$ can vary. For example, the unit-less term $[\mathbf{F}]_{1,1}$ is structured as

$$
\left(\frac{\sum_{\ell=0}^{2} g_{\ell, 2}(\theta, \phi) S_{\ell, 2-\ell}}{\lambda^{2}}\right)+\left(\frac{\sum_{\ell=0}^{3} g_{\ell, 3}(\theta, \phi) S_{\ell, 3-\ell}}{\lambda^{3}}\right) \epsilon+\left(\frac{\sum_{\ell=0}^{4} g_{\ell, 4}(\theta, \phi) S_{\ell, 4-\ell}}{\lambda^{4}}\right) \epsilon^{2}+o\left(\epsilon^{2}\right),
$$

where $o\left(\epsilon^{2}\right)$ gathers all the remaining term of $[\mathbf{F}]_{1,1}$ with $\lim _{\epsilon \rightarrow 0} o(\epsilon) / \epsilon=0$. Focusing on the $r$ dependence, $[\mathbf{F}]_{1,1}$ is thus structured as $b_{0}^{1,1}+b_{1}^{1,1} \epsilon+b_{2}^{1,1} \epsilon^{2}+o\left(\epsilon^{2}\right)$. Applying this same expansion methodology to all the terms of $\mathbf{F}(\boldsymbol{\alpha})$ ultimately, leads to the expression

$$
\mathbf{F}(\boldsymbol{\alpha})=\left[\begin{array}{ccc}
b_{0}^{1,1}+b_{1}^{1,1} \epsilon+b_{2}^{1,1} \epsilon^{2}+o\left(\epsilon^{2}\right) & b_{0}^{1,2}+b_{1}^{1,2} \epsilon+b_{2}^{1,2} \epsilon^{2}+o\left(\epsilon^{2}\right) & b_{2}^{1,3} \epsilon^{2}+b_{3}^{1,3} \epsilon^{3}+o\left(\epsilon^{3}\right) \\
b_{0}^{1,2}+b_{1}^{1,2} \epsilon+b_{2}^{1,2} \epsilon^{2}+o\left(\epsilon^{2}\right) & b_{0}^{2,2}+b_{1}^{2,2} \epsilon+b_{2}^{2,2} \epsilon^{2}+o\left(\epsilon^{2}\right) & b_{2}^{2,3} \epsilon^{2}+b_{3}^{2,3} \epsilon^{3}+o\left(\epsilon^{3}\right) \\
b_{2}^{1,3} \epsilon^{2}+b_{3}^{1,3} \epsilon^{3}+o\left(\epsilon^{3}\right) & b_{2}^{2,3} \epsilon^{2}+b_{3}^{2,3} \epsilon^{3}+o\left(\epsilon^{3}\right) & \epsilon^{4}\left(b_{4}^{3,3}+b_{5}^{3,3} \epsilon+b_{6}^{3,3} \epsilon^{2}+o\left(\epsilon^{2}\right)\right)
\end{array}\right] .
$$

We first calculate off-diagonal terms in order to identify decoupling conditions. After tedious algebraic manipulations, they are found to be given by

$$
\begin{aligned}
\frac{c}{r^{2} \sin \phi \cos \phi}[\mathbf{F}]_{1,2} & =P\left(\frac{S_{0,2}-S_{2,0}}{r^{2}} \sin \theta \cos \theta+\frac{S_{1,1}}{r^{2}} \cos 2 \theta\right)+o\left(\epsilon^{2}\right) \\
\frac{2 c}{r \sin \phi}[\mathbf{F}]_{1,3} & =P\left(\frac{S_{0,3}}{r^{3}} \cos \theta\left(1-\sin ^{2} \phi \sin ^{2} \theta\right)-\frac{S_{3,0}}{r^{3}} \sin \theta\left(1-\sin ^{2} \phi \cos ^{2} \theta\right)+\frac{S_{2,1}}{r^{3}} \cos \theta\right. \\
\left.\left(1-\sin ^{2} \phi \cos ^{2} \theta+2 \sin ^{2} \phi \sin ^{2} \theta\right)-\frac{S_{1,2}}{r^{3}} \sin \theta\left(1-\sin ^{2} \phi \sin ^{2} \theta+2 \sin ^{2} \phi \cos ^{2} \theta\right)\right)+o\left(\epsilon^{3}\right) & \\
\frac{2 c}{r \cos \phi}[\mathbf{F}]_{2,3} & =P\left(\frac{S_{0,3}}{r^{3}} \sin \theta\left(1-\sin ^{2} \phi \sin ^{2} \theta\right)+\frac{S_{3,0}}{r^{3}} \cos \theta\left(1-\sin ^{2} \phi \cos ^{2} \theta\right)\right. \\
& \left.+\frac{S_{2,1}}{r^{3}} \sin \theta\left(1-3 \sin ^{2} \phi \cos ^{2} \theta\right)+\frac{S_{1,2}}{r^{3}} \cos \theta\left(1-3 \sin ^{2} \phi \sin ^{2} \theta\right)\right)+o\left(\epsilon^{3}\right),
\end{aligned}
$$

where $c \stackrel{\text { def }}{=} \frac{\lambda^{2}}{4 \pi^{2} c_{\sigma}}$.

Second, we seek to decouple $\theta$ and $\phi$ to the zero order in $\epsilon$ (i.e., by imposing $b_{0}^{1,2}=0$ ) and to decouple $(\theta, \phi)$ and $r$ to the second order in $\epsilon$ (i.e., by imposing $b_{2}^{1,3}=b_{2}^{2,3}=0$ ). Equalizing $b_{0}^{1,2}$ to zero, i.e., the term $\left(\frac{S_{0,2}-S_{2,0}}{r^{2}} \sin \theta \cos \theta+\frac{S_{1,1}}{r^{2}} \cos 2 \theta\right)$ of (7) implies

$$
S_{1,1}=0 \text { and } S_{2,0}=S_{0,2}
$$

which concurs with far-field conditions given in [8] and [17] for which $\theta$ and $\phi$ estimation are both decoupled and isotropic (w.r.t. the azimuth $\theta$ ).

In the same way, both $b_{2}^{1,3}$ of (8) and $b_{2}^{2,3}$ of (9) are zero if $S_{0,3}=S_{1,2}=S_{2,1}=S_{3,0}=0$. Careful 
examination of $\mathbf{F}(\boldsymbol{\alpha})$ terms shows that these latter conditions also imply $b_{1}^{1,1}=b_{1}^{2,2}=b_{1}^{1,2}=0$. Ultimately, to ease the inversion of $\mathbf{F}(\boldsymbol{\alpha})$, we need $b_{5}^{3,3}=0$. The latter is satisfied under the additional conditions $S_{0,5}=$ $S_{1,4}=S_{2,3}=S_{3,2}=S_{4,1}=0$. All these conditions are simultaneously expressed by the following:

$$
S_{1,1}=0, S_{0,2}=S_{2,0} \text { and } S_{i, j}=0 \text { for } i+j=3,5 \text {. }
$$

We note that these conditions (11) which include the far-field conditions (10), are much more severe. For example the V-shaped antenna array highlighted in [8] satisfies (10) but no longer satisfies (11).

Under the conditions (11), (6) simplifies to

$$
\mathbf{F}(\boldsymbol{\alpha})=\left[\begin{array}{ccc}
b_{0}^{1,1}+b_{2}^{1,1} \epsilon^{2}+o\left(\epsilon^{2}\right) & b_{2}^{1,2} \epsilon^{2}+o\left(\epsilon^{2}\right) & b_{3}^{1,3} \epsilon^{3}+o\left(\epsilon^{3}\right) \\
b_{2}^{1,2} \epsilon^{2}+o\left(\epsilon^{2}\right) & b_{0}^{2,2}+b_{2}^{2,2} \epsilon^{2}+o\left(\epsilon^{2}\right) & b_{3}^{2,3} \epsilon^{3}+o\left(\epsilon^{3}\right) \\
b_{3}^{1,3} \epsilon^{3}+o\left(\epsilon^{3}\right) & b_{3}^{2,3} \epsilon^{3}+o\left(\epsilon^{3}\right) & \epsilon^{4}\left(b_{4}^{3,3}+b_{6}^{3,3} \epsilon^{2}+o\left(\epsilon^{2}\right)\right)
\end{array}\right],
$$

making it possible to obtain, after straightforward algebraic manipulations, the following expressions of the CRBs

$$
\begin{gathered}
\operatorname{CRB}(\theta)=\frac{1}{b_{0}^{1,1}}\left(1-\epsilon^{2}\left(\frac{b_{2}^{1,1}}{b_{0}^{1,1}}-\frac{\left(b_{3}^{1,3}\right)^{2}}{b_{0}^{1,1} b_{4}^{3,3}}\right)\right)+o\left(\epsilon^{2}\right) \\
\operatorname{CRB}(\phi)=\frac{1}{b_{0}^{2,2}}\left(1-\epsilon^{2}\left(\frac{b_{2}^{2,2}}{b_{0}^{2,2}}-\frac{\left(b_{3}^{2,3}\right)^{2}}{b_{0}^{2,2} b_{4}^{3,3}}\right)\right)+o\left(\epsilon^{2}\right) \\
\operatorname{CRB}(r)=\frac{1}{b_{4}^{3,3} \epsilon^{4}}\left(1-\epsilon^{2}\left(\frac{b_{6}^{3,3}}{b_{4}^{3,3}}-\frac{\left(b_{3}^{1,3}\right)^{2}}{b_{0}^{1,1} b_{4}^{3,3}}-\frac{\left(b_{3}^{2,3}\right)^{2}}{b_{0}^{2,2} b_{4}^{3,3}}\right)+o\left(\epsilon^{2}\right)\right) .
\end{gathered}
$$

The fact that

$$
\lim _{r \rightarrow \infty} \operatorname{CRB}(\theta)=\operatorname{CRB}_{\mathrm{FF}}(\theta)=\frac{1}{b_{0}^{1,1}} \text { and } \lim _{r \rightarrow \infty} \operatorname{CRB}(\phi)=\operatorname{CRB}_{\mathrm{FF}}(\phi)=\frac{1}{b_{0}^{2,2}},
$$

where $\mathrm{CRB}_{\mathrm{FF}}(\theta)$ and $\mathrm{CRB}_{\mathrm{FF}}(\phi)$ denote the far-field $\mathrm{CRBs}$, means that arrays satisfying conditions (11) do achieve the far-field CRBs when the source-to-array distance $r$ tends to infinity. In contrast, arrays that do not satisfy conditions (11) do not necessary satisfy (16) (see an example for linear arrays in [13]), an unexpected behavior due to a possible coupling $\left(b_{2}^{1,3} \neq 0, b_{2}^{2,3} \neq 0\right)$ between $(\theta, \phi)$ and $r$ in $\mathbf{F}(\boldsymbol{\alpha})$ to the second-order in $\epsilon$. Finally, for a source in the plane $(x, y)$, (15) reduces to

$$
\operatorname{CRB}(r)=\frac{1}{b_{4}^{3,3} \epsilon^{4}}\left(1-\epsilon^{2}\left(\frac{b_{6}^{3,3}}{b_{4}^{3,3}}-\frac{\left(b_{3}^{1,3}\right)^{2}}{b_{0}^{1,1} b_{4}^{3,3}}\right)+o\left(\epsilon^{2}\right)\right)_{\phi=\pi / 2} .
$$

\section{B. Special classes of arrays : Expressions of $\mathbf{F}(\boldsymbol{\alpha})$}

Conditions (11) are satisfied by many structured planar arrays. We study in details the following three classes of planar arrays for which the expression of $\mathbf{F}(\boldsymbol{\alpha})$ are derived, as well as expressions (13), (14) and (15) of the CRBs. 
1) Concentric uniform circular-based arrays: The $P$ sensors are divided into $I$ groups of respective sizes $P_{1}, \cdots, P_{I}$ where $\sum_{i=1}^{I} P_{i}=P$. The $i$-th group of sensors is placed uniformly along a circle of radius $r_{i}$ so that sensor $p_{i}, p_{i}=1, \ldots, P_{i}$ forms an angle $\theta_{p_{i}, i} \stackrel{\text { def }}{=} \theta_{i}+\theta-\frac{2 \pi\left(p_{i}-1\right)}{P_{i}}$ with $[O, x), \theta_{i}$ being an arbitrarily selected offset angle ${ }^{2}$. Parameter $\beta_{p}$ of the phase $\tau_{p}$ given in (1) can be expressed as

$$
\beta_{p}=1-\frac{2 r_{i}}{r} \cos \theta_{p_{i}, i} \sin \phi+\frac{r_{i}^{2}}{r^{2}}
$$

associated with a sensor on a circle of radius $r_{i}$. Using the identity

$$
\sum_{p_{i}=1}^{P_{i}} e^{i k \theta_{p_{i}, i}}=\left\{\begin{array}{ll}
P_{i} e^{i k \theta} & \text { if } k / P_{i} \in \mathbb{N} \\
0 & k \text { otherwise }
\end{array},\right.
$$

we easily prove that conditions (11) are satisfied if each circle include more than $5\left(P_{i}>5\right.$, for all $\left.i\right)$ sensors.

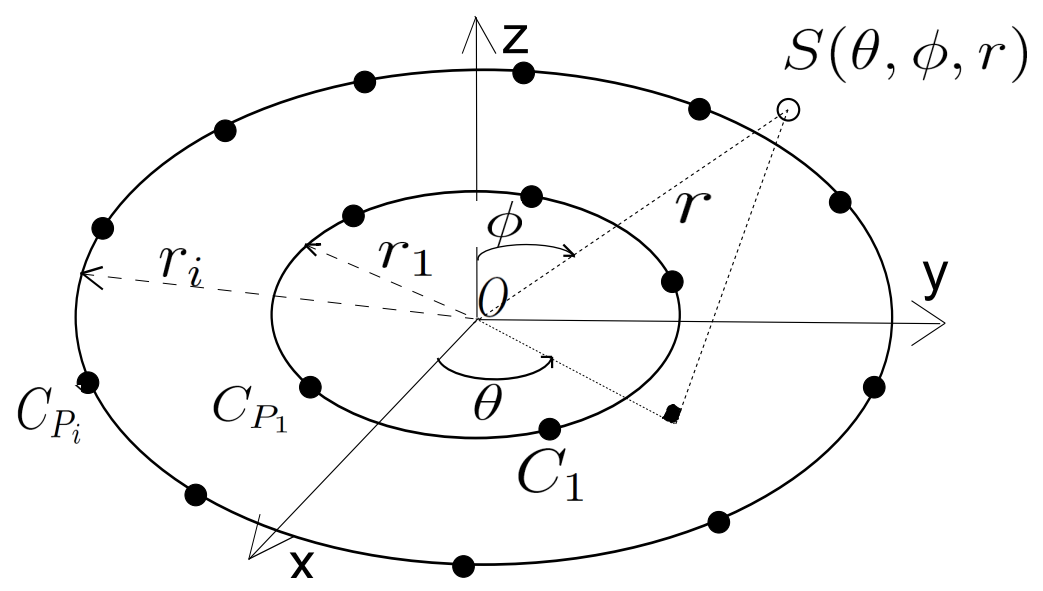

Fig.1 Concentric uniform circular-based array and source parameters.

By using the sensors polar coordinates $\left(r_{i}, \theta_{p_{i}, i}\right)$, the following Taylor expansions of the terms of the matrix

\footnotetext{
${ }^{2}$ These arrays are centro-symmetric, only if $\left(P_{i}\right)_{i=1, . ., I}$ are all even. They include as particular case, the so-called uniform concentric circular arrays [16] where $\theta_{i}=0$ and the number $P_{i}$ of sensors on each circle $C_{i}$ is constant.
} 
$\mathbf{F}(\boldsymbol{\alpha})$ are proved in the Appendix VI-B1 for $P_{i} \geq 6$ :

$$
\begin{aligned}
\frac{2 c}{r^{2} \sin ^{2} \phi}[\mathbf{F}]_{1,1} & =P \sum_{i=1}^{I} P_{i}\left(\frac{r_{i}^{2}}{r^{2}}-\frac{r_{i}^{4}}{r^{4}} \cos ^{2} \phi\right)+o\left(\epsilon^{4}\right) \\
\frac{2 c}{r^{2} \cos ^{2} \phi}[\mathbf{F}]_{2,2} & =P \sum_{i=1}^{I} P_{i}\left(\frac{r_{i}^{2}}{r^{2}}-\frac{r_{i}^{4}}{r^{4}}\left(1-3 \sin ^{2} \phi\right)\right)-\sum_{i=1}^{I} \frac{P_{i}^{2}}{2} \frac{r_{i}^{4}}{r^{4}} \sin ^{2} \phi-\sum_{i \neq j} P_{i} P_{j} \frac{r_{i}^{2} r_{j}^{2}}{r^{4}} \sin ^{2} \phi \\
& +o\left(\epsilon^{4}\right) \\
\frac{c}{r^{2} \sin ^{3} \phi \cos \phi}[\mathbf{F}]_{1,2} & =o\left(\epsilon^{4}\right) \\
\frac{c}{r \sin ^{4} \phi}[\mathbf{F}]_{1,3} & =o\left(\epsilon^{4}\right) \\
\frac{c}{r \cos \phi}[\mathbf{F}]_{2,3} & =P \sum_{i=1}^{I} \frac{P_{i}}{4} \frac{r_{i}^{4}}{r^{4}}\left(3-\frac{9}{4} \sin ^{2} \phi\right) \sin \phi-\sum_{i=1}^{I} \frac{P_{i}^{2}}{4} \frac{r_{i}^{4}}{r^{4}}\left(1+\frac{1}{2} \sin ^{2} \phi\right) \sin \phi \\
& -\frac{1}{4} \sum_{i \neq j} P_{j} \frac{r_{i}^{2} r_{j}^{2}}{r^{4}}\left(P_{j}-\frac{P_{i}}{2} \sin ^{2} \phi\right)+o\left(\epsilon^{4}\right) \\
c[\mathbf{F}]_{3,3} & =P \sum_{i=1}^{I} P_{i} \frac{r_{i}^{4}}{r^{4}} g_{1}\left(\sin ^{2} \phi\right)-\sum_{i=1}^{I} P_{i}^{2} \frac{r_{i}^{4}}{r^{4}} g_{2}\left(\sin ^{2} \phi\right) \\
& +P \sum_{i=1}^{I} P_{i} \frac{r_{i}^{6}}{r^{6}} g_{3}\left(\sin ^{2} \phi\right)-\sum_{i=1}^{I} P_{i}^{2} \frac{r_{i}^{6}}{r^{6}} g_{4}\left(\sin ^{2} \phi\right)-\sum_{i \neq j} P_{i} P_{j} \frac{r_{i}^{2} r_{j}^{4}}{r^{6}} g_{4}\left(\sin ^{2} \phi\right)+o\left(\epsilon^{4}\right)
\end{aligned}
$$

where $\epsilon \stackrel{\text { def }}{=} \frac{\max _{i}\left(r_{i}\right)}{r}$. Exact expressions of polynomials $g_{1}, g_{2}, g_{3}$ and $g_{4}$ are given in Appendix VI-B2.

2) Cross-based and square-based centro-symmetric arrays: For cross-based centro-symmetric arrays, as shown in Fig.2, sensors are placed along the $x$-axis and the $y$-axis, symmetrically around the origin i.e., at coordinates $\left( \pm a_{q}, 0\right)$ and $\left(0, \pm a_{q}\right)$, resulting in a total number of sensors $P=2 Q-1$ or $P=2 Q$ depending on whether a sensor is placed at the origin or not, where $Q$ is the number of sensors on each axis. We have $S_{i, j}=0$ for arbitrary $i \neq 0$ and $j \neq 0$, hence satisfying conditions (11). Non-zero geometric parameters $S_{i, j}$ are $\sum_{q=1}^{Q} a_{q}^{2}=S_{2,0}=S_{0,2} \stackrel{\text { def }}{=} \Sigma_{2}, \sum_{q=1}^{Q} a_{q}^{4}=S_{4,0}=S_{0,4} \stackrel{\text { def }}{=} \Sigma_{4}$ and $\sum_{q=1}^{Q} a_{q}^{6}=S_{6,0}=S_{0,6} \stackrel{\text { def }}{=} \Sigma_{6}$. 


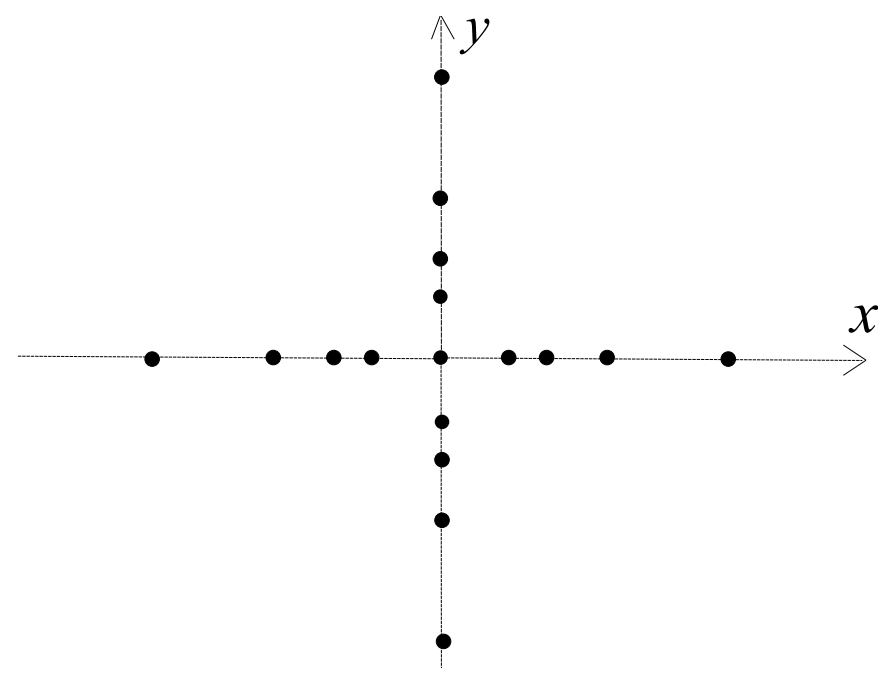

Fig.2 Cross-based centro-symmetric array.

Square-based centro-symmetric arrays shown in Fig.3 are made of $P=Q^{2}$ sensors at positions $\left(a_{q}, a_{q^{\prime}}\right)_{q=1, \ldots, Q, q^{\prime}=1, \ldots, Q}$ such that if a sensor is placed at some position $\left(x_{p}, y_{p}\right)$, another one is placed in the coordinate $\left(-x_{p},-y_{p}\right) . S_{i, j}$ are found to satisfy conditions (11). Non-zero ones reduce to $S_{2,0}=S_{0,2} \stackrel{\text { def }}{=} Q \Sigma_{2}$, $S_{4,0}=S_{0,4} \stackrel{\text { def }}{=} Q \Sigma_{4}, S_{6,0}=S_{0,6} \stackrel{\text { def }}{=} Q \Sigma_{6}, S_{2,2}=\Sigma_{2}^{2}$, and $S_{4,2}=S_{2,4}=\Sigma_{2} \Sigma_{4}$, where $\Sigma_{2}, \Sigma_{4}$ and $\Sigma_{6}$ have the same definition as for the cross-based arrays.

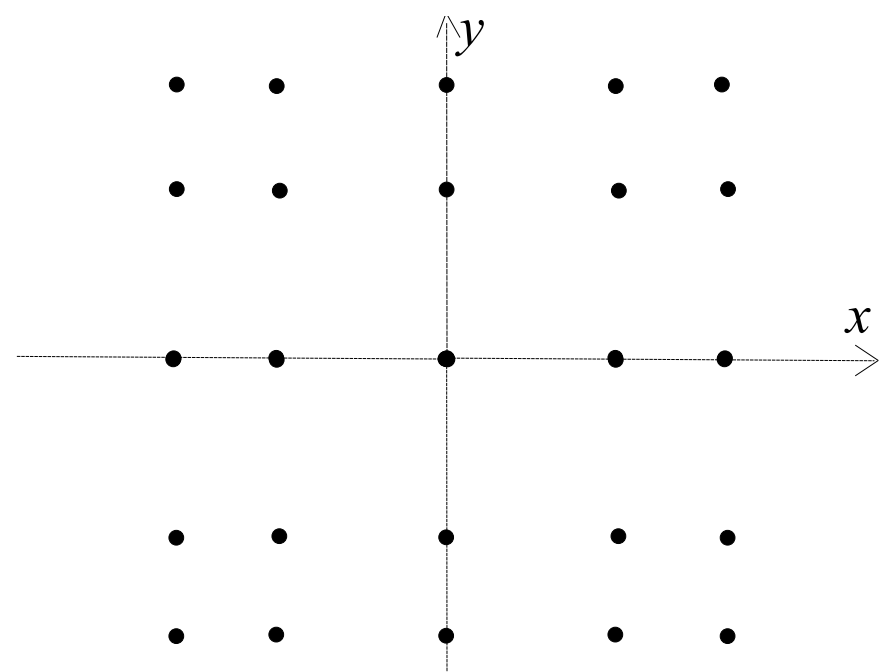

Fig.3 Square-based centro-symmetric array.

For these two (cross and square based) classes of centro-symmetric arrays, we reach the following unified 
expression ${ }^{3}$ of the Taylor expansion of the matrix $\mathbf{F}(\boldsymbol{\alpha})$, proved in the Appendix VI-C:

$$
\begin{aligned}
\frac{c}{r^{2} \sin ^{2} \phi}[\mathbf{F}]_{1,1} & =\frac{a_{2}^{1,1} \Sigma_{2}}{r^{2}}+\frac{a_{4}^{1,1}(\theta, \phi) Q \Sigma_{4}+a_{2^{2}}^{1,1}(\theta, \phi) \Sigma_{2}^{2}}{r^{4}}+o\left(\epsilon^{4}\right) \\
\frac{c}{r^{2} \cos ^{2} \phi}[\mathbf{F}]_{2,2} & =\frac{a_{2}^{2,2} \Sigma_{2}}{r^{2}}+\frac{a_{4}^{2,2}(\theta, \phi) Q \Sigma_{4}+a_{2^{2}}^{2,2}(\theta, \phi) \Sigma_{2}^{2}}{r^{4}}+o\left(\epsilon^{4}\right) \\
\frac{c}{r^{2} \sin ^{3} \phi \cos \phi}[\mathbf{F}]_{1,2} & =\frac{a_{4}^{1,2}(\theta, \phi) Q \Sigma_{4}+a_{2^{2}}^{1,2}(\theta, \phi) \Sigma_{2}^{2}}{r^{4}}+o\left(\epsilon^{4}\right) \\
\frac{c}{r \sin ^{4} \phi}[\mathbf{F}]_{1,3} & =\frac{a_{4}^{1,3}(\theta, \phi) Q \Sigma_{4}+a_{2^{2}}^{1,3}(\theta, \phi) \Sigma_{2}^{2}}{r^{4}}+o\left(\epsilon^{4}\right) \\
\frac{c}{r \sin \phi \cos \phi}[\mathbf{F}]_{2,3} & =\frac{a_{4}^{2,3}(\theta, \phi) Q \Sigma_{4}+a_{2^{2}}^{2,3}(\theta, \phi) \Sigma_{2}^{2}}{r^{4}}+o\left(\epsilon^{4}\right) \\
c[\mathbf{F}]_{3,3} & =\frac{a_{4}^{3,3}(\theta, \phi) Q \Sigma_{4}+a_{2^{2}}^{3,3}(\theta, \phi) \Sigma_{2}^{2}}{r^{4}} \\
& +\frac{a_{6}^{3,3}(\theta, \phi) Q^{2} \Sigma_{6}+a_{2,4}^{3,3}(\theta, \phi) Q \Sigma_{2} \Sigma_{4}+a_{2^{3}}^{3,3}(\theta, \phi) \Sigma_{2}^{3}}{r^{6}}+o\left(\epsilon^{6}\right) .
\end{aligned}
$$

where $a_{2}^{1,1}=a_{2}^{2,2}=P$ [resp., $P Q$ ] for the cross-based [resp., square-based] centro-symmetric arrays. Expressions of $a_{k}^{i, j}(\theta, \phi)$, given in Appendix VI-C, are functions of the number of sensors and $(\theta, \phi)$. Also, $a_{2^{2}}^{1,1}(\theta, \phi)=a_{2^{2}}^{1,2}(\theta, \phi)=a_{2^{2}}^{1,3}(\theta, \phi)=a_{2^{3}}^{3,3}(\theta, \phi)=0$ for the cross-based centro-symmetric arrays.

\section{Special classes of arrays : Expressions of the CRBs}

1) Concentric uniform circular-based arrays: Using (13) and the values of the parameters $b_{k}^{i, j}$ of the matrix $\mathbf{F}(\boldsymbol{\alpha})$ of (12) derived by identification with the expansion (20)-(25), we deduce the following closed-form expression of the CRB on the azimuth:

$$
\operatorname{CRB}(\theta)=\operatorname{CRB}_{\mathrm{FF}}(\theta)\left(1+\frac{\sum_{i=1}^{I} P_{i} r_{i}^{4} \cos ^{2} \phi}{r^{2} \sum_{i=1}^{I} P_{i} r_{i}^{2}}+o\left(\epsilon^{2}\right)\right),
$$

where we obtain the following original expression of the CRB on the azimuth under the far-field conditions,

$$
\mathrm{CRB}_{\mathrm{FF}}(\theta)=\frac{2 c}{\sin ^{2} \phi} \frac{1}{P \sum_{i=1}^{I} P_{i} r_{i}^{2}} \text {. }
$$

For a single-ring UCA of radius $r_{1}$, Eq. (32) simplifies to

$$
\operatorname{CRB}(\theta)=\operatorname{CRB}_{\mathrm{FF}}(\theta)\left(1+\frac{r_{1}^{2}}{r^{2}} \cos ^{2} \phi+o\left(\frac{r_{1}^{2}}{r^{2}}\right)\right)
$$

with $\operatorname{CRB}_{\mathrm{FF}}(\theta)=\frac{2 c}{\sin ^{2} \phi} \frac{1}{P^{2} r_{1}^{2}}$.

Expressions of the CRB on the elevation and range deduced from (14) and (15) are much more intricate. Consequently, we concentrate on the single-ring UCA for which we obtain the following closed-form

\footnotetext{
${ }^{3}$ Note that the number $Q$ introduced in some terms will allow us to obtain the common expressions of the CRB (42), (43) and (44).
} 
expressions:

$$
\begin{aligned}
\operatorname{CRB}(\phi) & =\operatorname{CRB}_{\mathrm{FF}}(\phi)\left[1+\frac{r_{1}^{2}}{r^{2}} h_{2}\left(\sin ^{2} \phi\right)+o\left(\frac{r_{1}^{2}}{r^{2}}\right)\right] \\
\operatorname{CRB}(r) & =\frac{32 c}{\sin ^{4} \phi} \frac{r^{4}}{r_{1}^{4}}\left[1+\frac{r_{1}^{2}}{r^{2}} h_{3}\left(\sin ^{2} \phi\right)+o\left(\frac{r_{1}^{2}}{r^{2}}\right)\right],
\end{aligned}
$$

with $\operatorname{CRB}_{\mathrm{FF}}(\phi)=\frac{2 c}{\cos ^{2} \phi} \frac{1}{P^{2} r_{1}^{2}}$ and

$$
h_{2}\left(\sin ^{2} \phi\right) \stackrel{\text { def }}{=} \frac{16}{\sin ^{2} \phi}+\frac{39}{4} \sin ^{2} \phi-27 \text { and } h_{3}\left(\sin ^{2} \phi\right) \stackrel{\text { def }}{=} 5-\frac{21}{4} \sin ^{2} \phi .
$$

Further simplification are obtained for a single-ring UCA made of $P>8$ sensors, for which (20), (22) and (23) become:

$$
\begin{aligned}
\frac{2 c}{r^{2} \sin ^{2} \phi}[\mathbf{F}]_{1,1} & =P^{2}\left(\frac{r_{1}^{2}}{r^{2}}-\frac{r_{1}^{4}}{r^{4}} \cos ^{2} \phi+\frac{r_{1}^{4}}{r^{4}} g_{5}\left(\sin ^{2} \phi\right)+\frac{r_{1}^{6}}{r^{6}} g_{6}\left(\sin ^{2} \phi\right)\right)+o\left(\frac{r_{1}^{7}}{r^{7}}\right) \\
\frac{c}{r^{2} \sin \phi \cos \phi}[\mathbf{F}]_{1,2} & =o\left(\frac{r_{1}^{7}}{r^{7}}\right) \\
\frac{c}{r \sin \phi}[\mathbf{F}]_{1,3} & =o\left(\frac{r_{1}^{7}}{r^{7}}\right),
\end{aligned}
$$

with

$$
g_{5}\left(\sin ^{2} \phi\right) \stackrel{\text { def }}{=} 1-3 \sin ^{2} \phi+2 \sin ^{4} \phi \text { and } g_{6}\left(\sin ^{2} \phi\right) \stackrel{\text { def }}{=}-1+6 \sin ^{2} \phi-10 \sin ^{4} \phi+5 \sin ^{6} \phi .
$$

This allows us to further develop the Taylor expansion in (34) to obtain the following more accurate closed-form expression:

$$
\operatorname{CRB}(\theta)=\operatorname{CRB}_{\mathrm{FF}}(\theta)\left[1+\frac{r_{1}^{2}}{r^{2}} \cos ^{2} \phi+\frac{r_{1}^{4}}{r^{4}} \sin ^{2} \phi \cos ^{2} \phi+\frac{r_{1}^{6}}{r^{6}} h_{1}\left(\sin ^{2} \phi\right)+o\left(\frac{r_{1}^{7}}{r^{7}}\right)\right],
$$

with $h_{1}\left(\sin ^{2} \phi\right) \stackrel{\text { def }}{=}-\sin ^{2} \phi+3 \sin ^{4} \phi-2 \sin ^{6} \phi$. Interestingly, for a source in the $(x, y)$ plane (i.e., $\left.\phi=\pi / 2\right)$, we deduce from the matrix $\mathbf{F}(\boldsymbol{\alpha})$ that (40) and (17) give

$$
\begin{aligned}
& \operatorname{CRB}(\theta)=\operatorname{CRB}_{\mathrm{FF}}(\theta)\left[1+o\left(\frac{r_{1}^{7}}{r^{7}}\right)\right] \\
& \operatorname{CRB}(r)=\frac{32 c}{\sin ^{4} \phi} \frac{r^{4}}{r_{1}^{4}}\left[1-\frac{r_{1}^{2}}{2 r^{2}}+o\left(\frac{r_{1}^{2}}{r^{2}}\right)\right] .
\end{aligned}
$$

The validity of some approximate closed-form expressions of the CRB is illustrated for a source located with an azimuth $\theta=70^{\circ}$ and elevation $\phi=70^{\circ}$. Figs.4 and 5 compare the approximate ratios $\mathrm{CRB}(\theta) / \mathrm{CRB}_{\mathrm{FF}}(\theta)$ and $\operatorname{CRB}(\phi) / \mathrm{CRB}_{\mathrm{FF}}(\phi)$ given by (40) and (35) to the exact ones (i.e., derived from the numerical inversion of the matrix $\mathbf{F}(\boldsymbol{\alpha})$ built on the exact model of the time delay (1)). These figures naturally show that $\operatorname{CRB}(\theta)$ and $\operatorname{CRB}(\phi)$ tend to $\mathrm{CRB}_{\mathrm{FF}}(\theta)$ and $\operatorname{CRB}_{\mathrm{FF}}(\phi)$, respectively, when the range increases. In addition, we can notice that the far-field state is reached from the ratio $r / r_{1}=10$. We also see that the near-field CRB on the 
azimuth and elevation are smaller that the associated far-field CRB. We also consider Fig.6 that compares the approximate $\operatorname{CRB}(r)$ (36) to the exact one as a function of $r / r_{1}$. Figs.4 and 6 show that the approximate values of $\mathrm{CRB}$ on the azimuth and range are very close to the exact ones for 10 sensors from $r / r_{1}=2$. This contrasts with elevation for which the approximate values of the CRB are close to the exact one only from $r / r_{1}=4$. For 7 sensors, we note that our proposed approximations of all CRBs are still accurate from $r / r_{1}=4$.

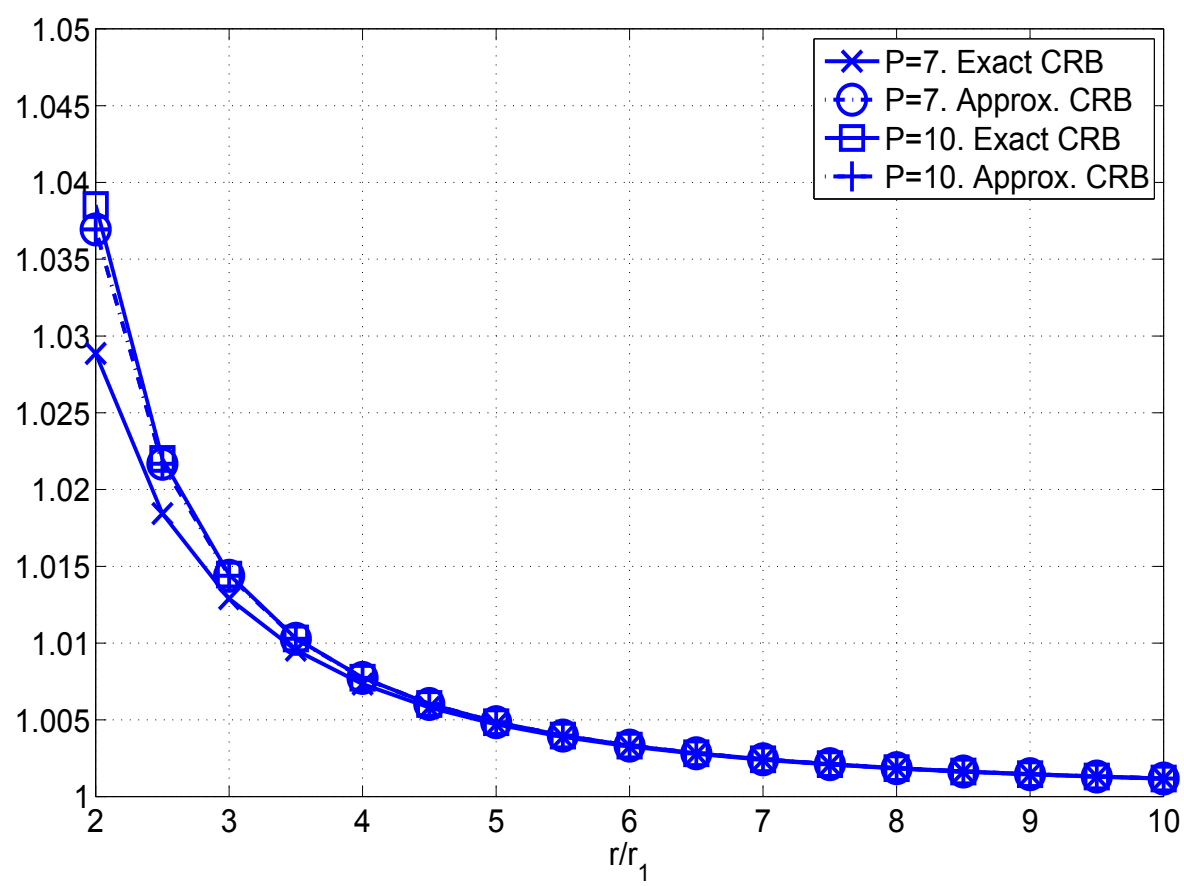

Fig.4 Approximate and exact ratios $\operatorname{CRB}(\theta) / \mathrm{CRB}_{\mathrm{FF}}(\theta)$ for the UCA. 


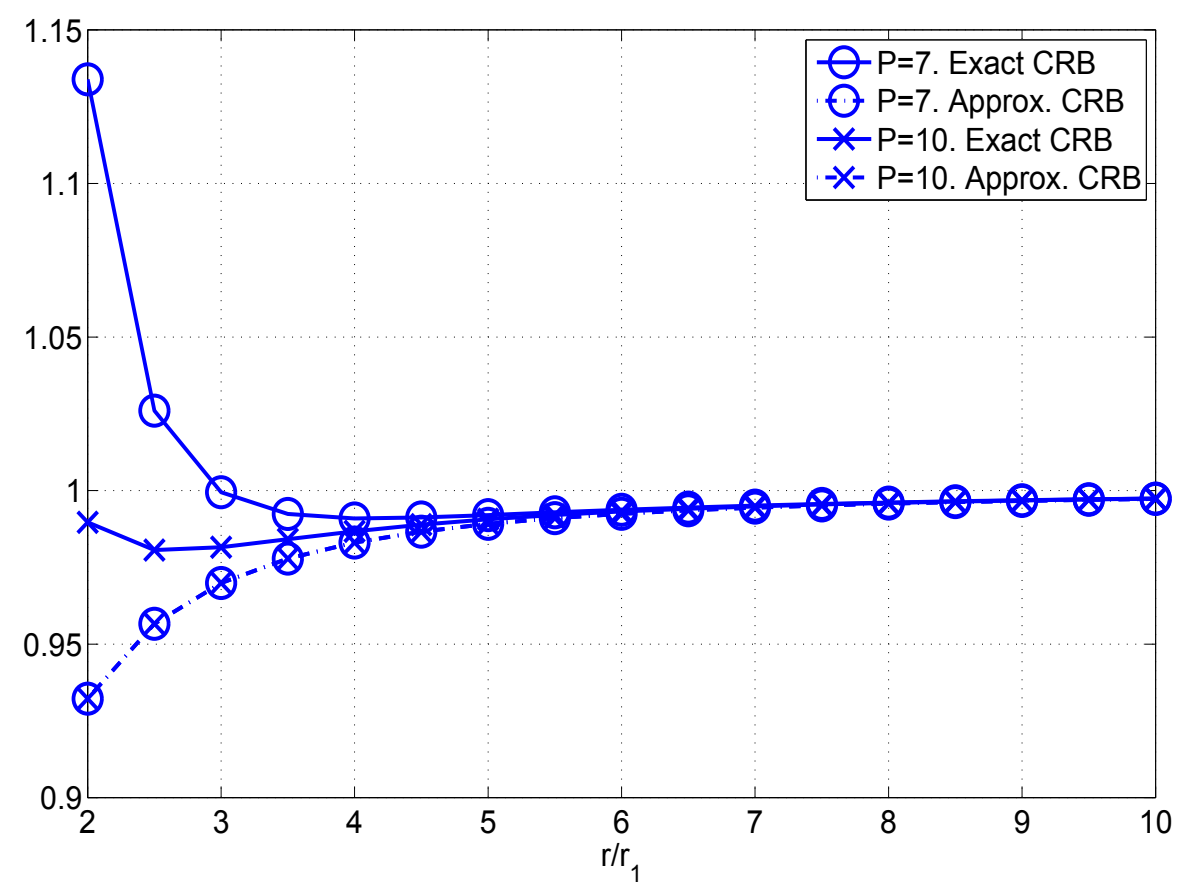

Fig.5 Approximate and exact ratios $\mathrm{CRB}(\phi) / \mathrm{CRB}_{\mathrm{FF}}(\phi)$ for the UCA.

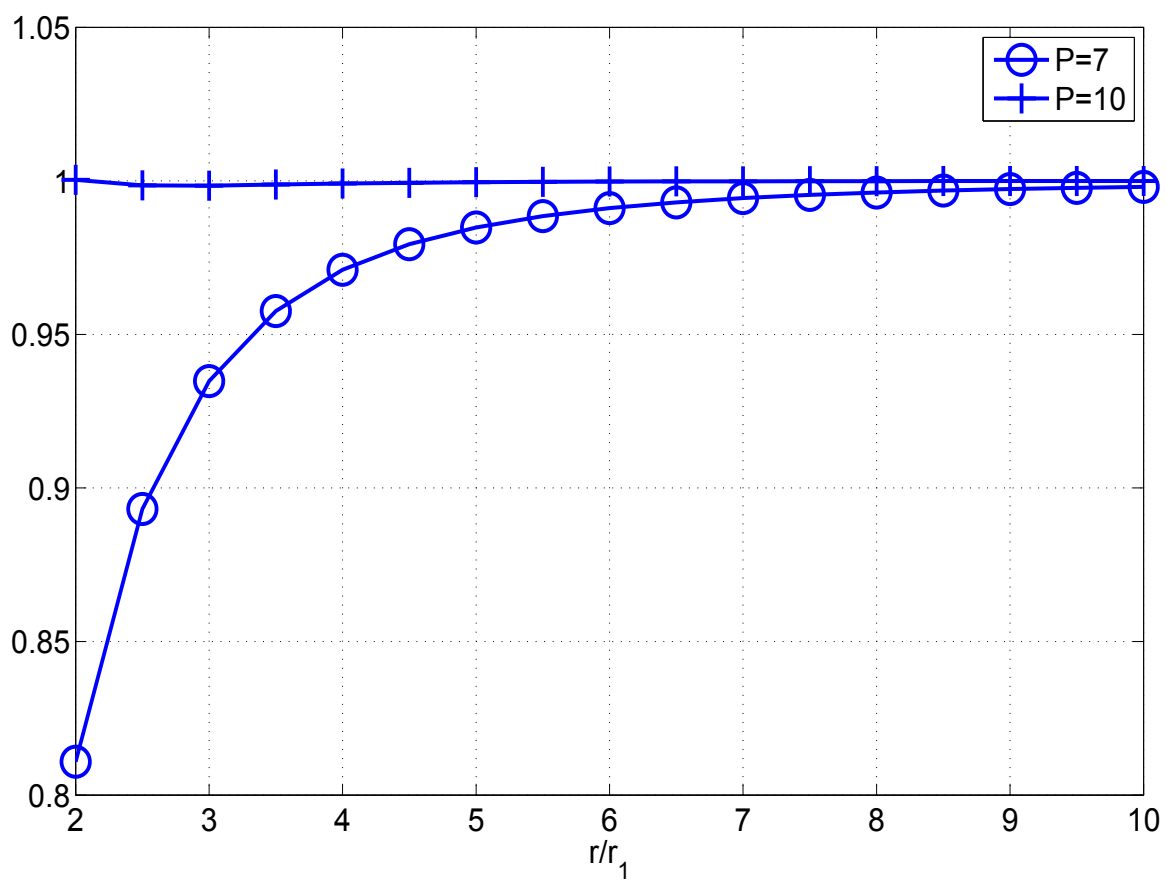

Fig.6 Ratio of the approximate $\operatorname{CRB}(r)$ to the exact one for the UCA.

2) Cross-based and square-based centro-symmetric arrays: For such arrays, (13), (14) and (15) give very intricate expressions. But hopefully, we can identify two geometric parameters $\kappa$ and $\eta$ that determine the near-field accuracy of the antenna array. They are defined by the following two unit-less array geometric 
expressions:

$$
\kappa \stackrel{\text { def }}{=} \frac{\Sigma_{2}^{2}}{Q \Sigma_{4}} \text { and } \eta \stackrel{\text { def }}{=} \frac{\Sigma_{2}^{3}}{Q^{2} \Sigma_{6}} .
$$

We note that they verify $0<\eta \leq \kappa \leq 1$ [12], and remain unchanged if a sensor is added/removed at/from the origin and if sensor coordinates are scaled by some arbitrary constant. The interest of these two parameters is that they complement the geometric parameter $\Sigma_{2}$ to characterize the behavior of the three CRBs in the near-field condition, allowing us to derive some optimizations. This contrasts with the far-field conditions for which $\Sigma_{2}$ characterizes the behavior of the the CRB on the azimuth and elevation in the near-field condition, (see (41)).

After tedious algebraic manipulations, CRBs (13), (14) and (15) can be rewritten in terms of these parameters, to obtain the following expressions:

$$
\begin{aligned}
& \operatorname{CRB}(\theta)=\operatorname{CRB}_{\mathrm{FF}}(\theta)\left(1+\frac{a(\theta, \phi, \kappa) \Sigma_{2}}{r^{2}}+o\left(\epsilon^{2}\right)\right) \\
& \operatorname{CRB}(\phi)=\operatorname{CRB}_{\mathrm{FF}}(\phi)\left(1+\frac{b(\theta, \phi, \kappa) \Sigma_{2}}{r^{2}}+o\left(\epsilon^{2}\right)\right) \\
& \operatorname{CRB}(r)=\frac{r^{4}}{d(\theta, \phi, \kappa) \Sigma_{2}^{2}}\left(1+\frac{e(\theta, \phi, \kappa, \eta) \Sigma_{2}}{r^{2}}+o\left(\epsilon^{2}\right)\right),
\end{aligned}
$$

where the far-field CRB on $\theta$ and $\phi$ are given, respectively, by

$$
\operatorname{CRB}_{\mathrm{FF}}(\theta)=\frac{c}{a_{2}^{1,1} \Sigma_{2} \sin ^{2} \phi} \text { and } \operatorname{CRB}_{\mathrm{FF}}(\phi)=\frac{c}{a_{2}^{2,2} \Sigma_{2} \cos ^{2} \phi},
$$

in which $a_{2}^{1,1}=a_{2}^{2,2}=P$ [resp., $\left.P Q\right]$ for the cross-based centro-symmetric arrays [resp., square-based centrosymmetric arrays] and

$$
\begin{aligned}
a(\theta, \phi, \kappa) & =\frac{\left(a_{4}^{1,3}(\theta, \phi)+\kappa a_{2^{2}}^{1,3}(\theta, \phi)\right)^{2} \sin ^{6} \phi-\left(a_{4}^{1,1}(\theta, \phi)+\kappa a_{2^{2}}^{1,1}(\theta, \phi)\right)\left(a_{4}^{3,3}(\theta, \phi)+\kappa a_{2^{2}}^{3,3}(\theta, \phi)\right)}{\kappa a_{2}^{1,1}\left(a_{4}^{3,3}(\theta, \phi)+\kappa a_{2^{2}}^{3,3}(\theta, \phi)\right)} \\
b(\theta, \phi, \kappa) & =\frac{\left(a_{4}^{2,3}(\theta, \phi)+\kappa a_{2^{2}}^{2,3}(\theta, \phi)\right)^{2} \sin ^{2} \phi-\left(a_{4}^{2,2}(\theta, \phi)+\kappa a_{2^{2}}^{2,2}(\theta, \phi)\right)\left(a_{4}^{3,3}(\theta, \phi)+\kappa a_{2^{2}}^{3,3}(\theta, \phi)\right)}{\kappa a_{2}^{2,2}\left(a_{4}^{3,3}(\theta, \phi)+\kappa a_{2^{2}}^{3,3}(\theta, \phi)\right)} \\
d(\theta, \phi, \kappa) & =\frac{1}{c}\left(\frac{1}{\kappa} a_{4}^{3,3}(\theta, \phi)+a_{2^{2}}^{3,3}(\theta, \phi)\right) \\
e(\theta, \phi, \kappa, \nu) & =\frac{\left(a_{4}^{1,3}(\theta, \phi)+\kappa a_{2^{2}}^{1,3}(\theta, \phi)\right)^{2} \sin ^{6} \phi}{\kappa a_{2}^{1,1}\left(a_{4}^{3,3}(\theta, \phi)+\kappa a_{2^{2}}^{3,3}(\theta, \phi)\right)}+\frac{\left(a_{4}^{2,3}(\theta, \phi)+\kappa a_{2^{2}}^{2,3}(\theta, \phi)\right)^{2} \sin ^{2} \phi}{\kappa a_{2}^{2,2}\left(a_{4}^{3,3}(\theta, \phi)+\kappa a_{2^{2}}^{3,3}(\theta, \phi)\right)} \\
& -\frac{\eta^{-1} a_{6}^{3,3}(\theta, \phi)+\kappa^{-1} a_{2,4}^{3,3}(\theta, \phi)+a_{2^{3}}^{3,3}(\theta, \phi)}{\kappa^{-1} a_{4}^{3,3}(\theta, \phi)+a_{2^{2}}^{3,3}(\theta, \phi)},
\end{aligned}
$$

where the terms $a_{k}^{i, j}(\theta, \phi)$ come from (26)-(31).

The validity of some approximate closed-form expressions of the CRB for the square-based centro-symmetric 
arrays $^{4}$ is illustrated for a source located with an azimuth $\theta=60^{\circ}$ and elevation $\phi=40^{\circ}$ for the specific case of uniform square-based arrays with half-wavelength inter-sensors spacing. As in the UCA case, Fig.7 and Fig.8 compare the approximate ratios $\operatorname{CRB}(\theta) / \mathrm{CRB}_{\mathrm{FF}}(\theta)$ and $\operatorname{CRB}(\phi) / \mathrm{CRB}_{\mathrm{FF}}(\phi)$ given by (42) and (43) to the exact ones. Fig.9 compares the approximate $\operatorname{CRB}(r)$, given by (44), to the exact one as a function of $r / r_{0}$ where $r_{0}$ is the half aperture $\frac{Q-1}{2} \frac{\lambda}{2}$ (similarly as $r / r_{1}$ for the UCA). The above figures confirm the validity of the proposed approximations for a large enough $Q$ and/or a large enough ratio $r / r_{0}$.

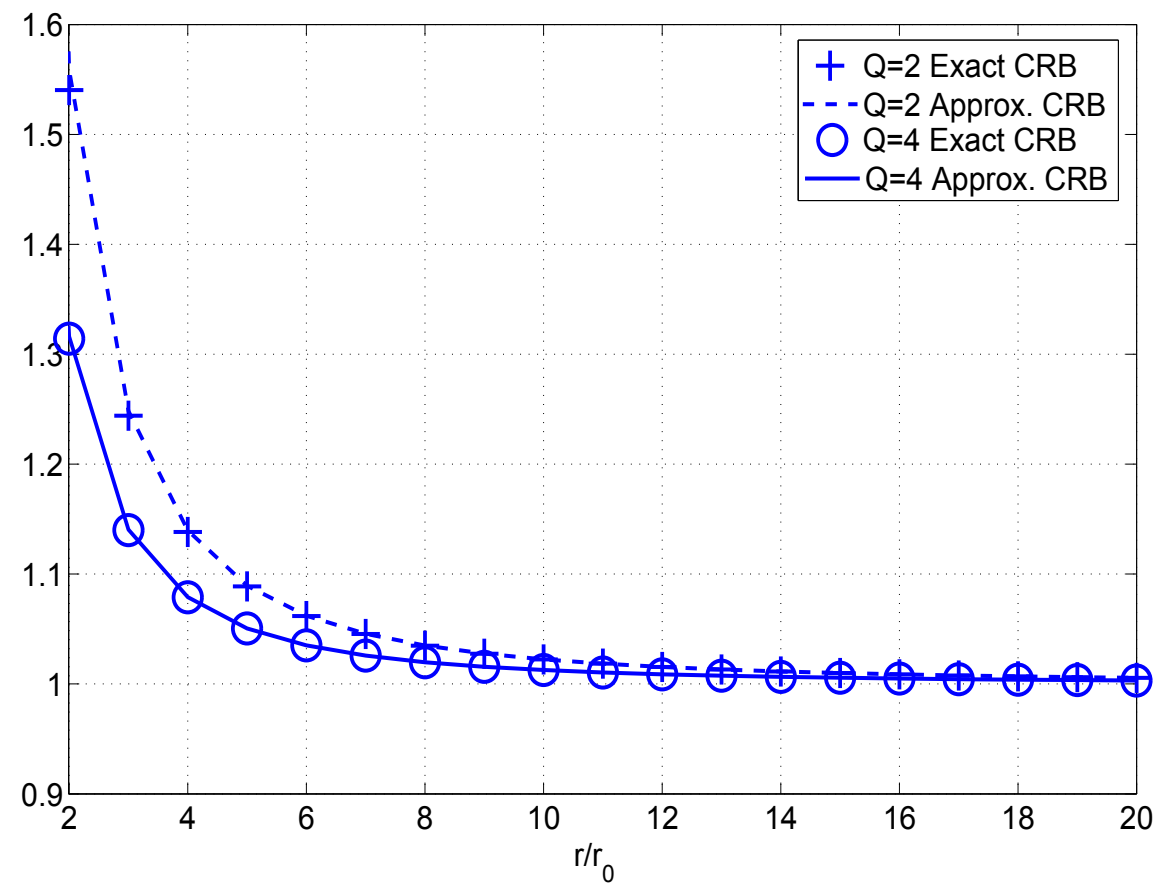

Fig.7 Approximate and exact ratios $\mathrm{CRB}(\theta) / \mathrm{CRB}_{\mathrm{FF}}(\theta)$ for uniform square-based arrays.

\footnotetext{
${ }^{4}$ The same behavior is noticed for the cross-based centro-symmetric arrays.
} 


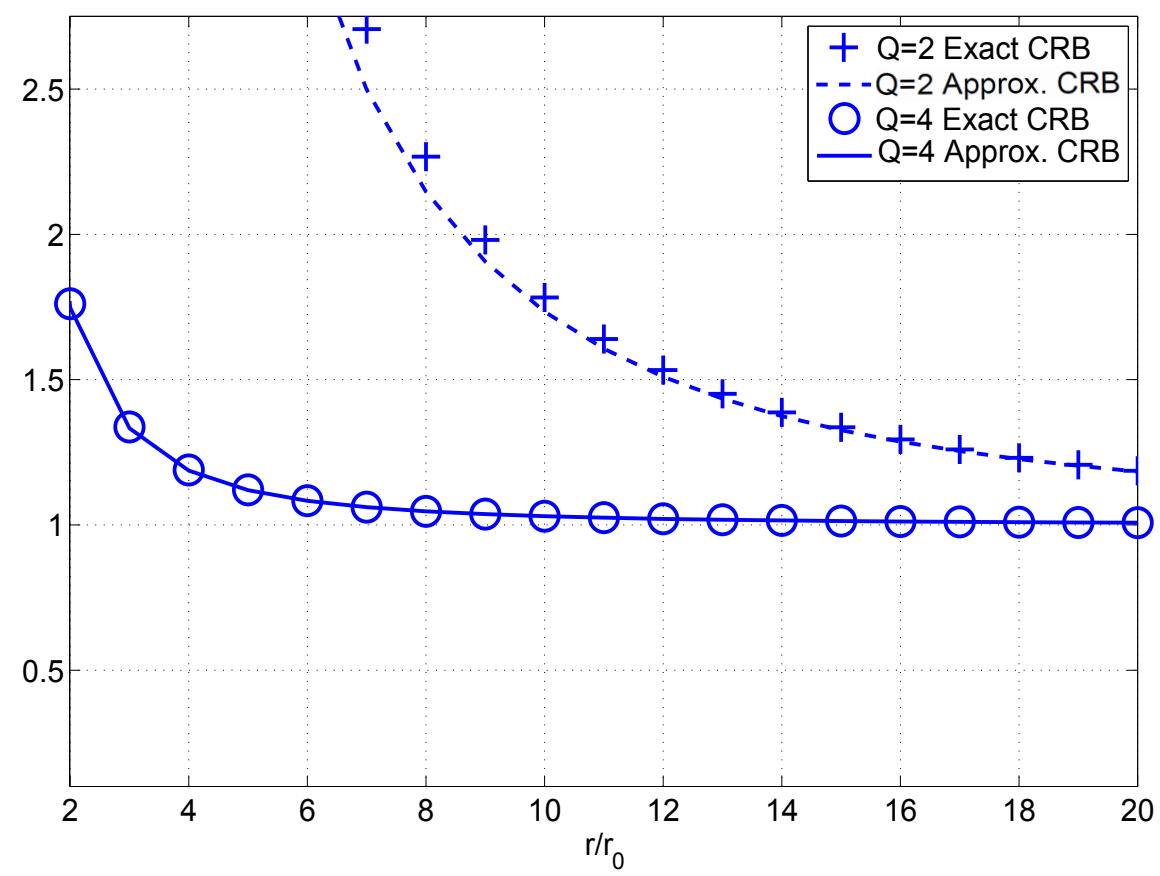

Fig.8 Approximate and exact ratios $\mathrm{CRB}(\phi) / \mathrm{CRB}_{\mathrm{FF}}(\phi)$ for uniform square-based arrays.

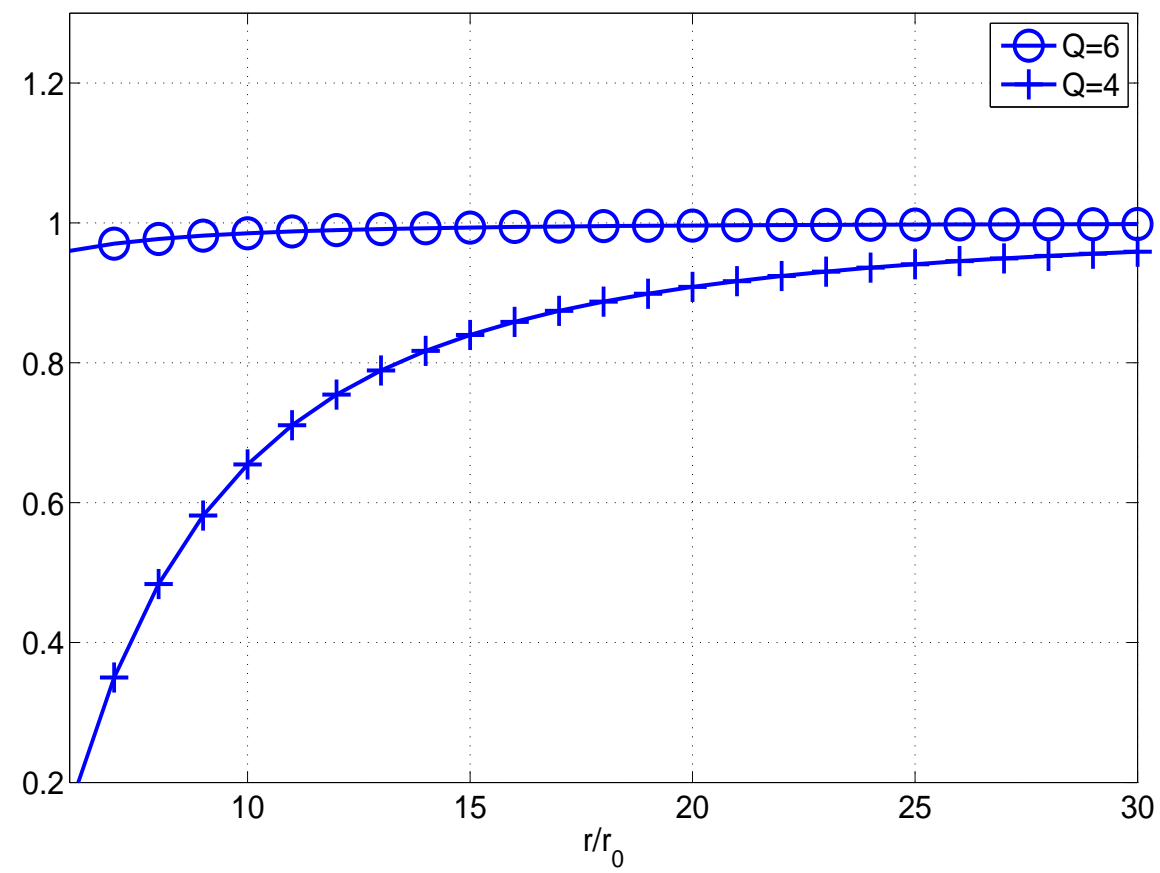

Fig.9 Ratio of the approximate $\operatorname{CRB}(r)$ to the exact one for uniform square-based arrays.

\section{ANALYSIS OF THE DERIVED CRBS}

A. Isotropy under the near-field

An antenna array appears to be isotropic to a source located in its far-field when sensors placed such that

$$
S_{1,1}=0 \text { and } S_{0,2}=S_{2,0} .
$$


This condition is weaker than conditions (11). Consequently, the isotropy property deteriorates if the source tends to be in the antenna near-field. For example, for cross-based and square-based centro-symmetric arrays, the CRBs on azimuth (42) and elevation (43) depend on the azimuth angle to the second-order in $\epsilon$, whereas for the CRB on the range (44), the dominant term is dependent on the azimuth. Furthermore, from expressions of $a_{k}^{i, j}(\theta, \phi)$ in Sec. VI-C, azimuth, elevation and range CRBs appear to be periodic in $\theta$ of period $\pi / 2$, as one may expect. Due to the intricate expressions of these CRBs, it is difficult to learn more about the deterioration of isotropy when the source range $r$ decreases or when the number of sensors $P$ decreases.

However, more can be learnt about single-ring UCA. First, thanks to (19), CRBs are periodic in $\theta$ of period $2 \pi / P$, as one may predict. Also if we denote the radius by $r_{1}$ and $\theta_{p, 1}$ by $\theta_{p}$, Taylor expansion of the elements of $\mathbf{F}(\boldsymbol{\alpha})$ w.r.t. $\epsilon$ (5), where only the $\theta$ dependence is retained, yields to

$$
[\mathbf{F}]_{i, j}=\sum_{k=0}^{\infty}\left(\sum_{p=1}^{P} g_{k}^{(i, j)}\left(\cos \theta_{p}, \sin \theta_{p}\right)\right) \epsilon^{k},
$$

where $g_{k}^{(i, j)}$ is a polynomial expression of $\cos \theta_{p}$ and $\sin \theta_{p}$ of degree $k+2, k+1$ or $k$ for $(i, j=1,2)$, $(i=1,2, j=3)$ or $(i=j=3)$, respectively. By linearizing this polynomial, we have for example for $i, j=1,2$ :

$$
g_{k}^{(1,2)}\left(\cos \theta_{p}, \sin \theta_{p}\right)=\sum_{\ell=0}^{k+2} c_{\ell, k}^{(1,2)} \cos \left(\ell \theta_{p}\right)+\sum_{\ell=1}^{k+2} s_{\ell, k}^{(1,2)} \sin \left(\ell \theta_{p}\right)
$$

where $c_{0, k}^{(1,2)}=0$ for odd degrees of $g_{k}^{(1,2)}$. Then, using (19), focusing on $\theta$ and carefully studying the first terms of the Taylor expansion (47) in $\epsilon$, we obtain

$$
\begin{aligned}
& {[\mathbf{F}]_{i, j}=\sum_{k=0}^{\lfloor(P-3) / 2\rfloor} b_{2 k}^{i, j} \epsilon^{2 k}+\sum_{k=P-2}^{\infty} b_{k}^{i, j}(\theta) \epsilon^{k}} \\
& {[\mathbf{F}]_{i, j}=\sum_{k=P-2}^{\infty} b_{k}^{i, j}(\theta) \epsilon^{k}} \\
& {[\mathbf{F}]_{2,3}=\sum_{k=3}^{P-1} b_{k}^{i, j} \epsilon^{k}+\sum_{k=P}^{\infty} b_{k}^{i, j}(\theta) \epsilon^{k}} \\
& {[\mathbf{F}]_{3,3}=\sum_{k=2}^{\lfloor(P-1) / 2\rfloor} b_{2 k}^{i, j} \epsilon^{2 k}+\sum_{k=P}^{\infty} b_{k}^{i, j}(\theta) \epsilon^{k},}
\end{aligned}
$$

for $P \geq 4$ and $i=j=1$ or $i=j=2(48), i=1, j=2,3$ (49), where $b_{k}^{i, j}$ and $b_{2 k}^{i, j}$ do not depend on $\theta$. For example, $\frac{2 c[\mathbf{F}]_{1,1}}{r_{0}^{2} \sin ^{2} \phi}$ is given in Table 1 for $P=3,4,5,6$ :

\begin{tabular}{|l|c|}
\hline$P$ & \\
\hline 3 & $1-\epsilon^{2} \sin \phi \cos 3 \theta+o\left(\epsilon^{2}\right)$ \\
\hline 4 & $1-\epsilon^{2}\left(\cos ^{2} \phi-\sin ^{2} \phi \sin 4 \theta\right)+o\left(\epsilon^{2}\right)$ \\
\hline 5 & $1-\epsilon^{2} \cos ^{2} \phi-\epsilon^{3} \sin ^{3} \phi \cos 5 \theta+o\left(\epsilon^{3}\right)$ \\
\hline 6 & $1-\epsilon^{2} \cos ^{2} \phi-\epsilon^{4}\left(1-3 \sin ^{2} \phi+2 \sin ^{4} \phi-\sin ^{4} \phi \cos 6 \theta\right)+o\left(\epsilon^{4}\right)$ \\
\hline
\end{tabular}


Table 1 Second-order expansion of $\frac{2 c[\mathbf{F}]_{1,1}}{r_{0}^{2} \sin ^{2} \phi}$ for for $P=3,4,5,6$.

The following can be concluded about a single ring UCA of a fixed number $P$ of sensors: From (48)-(51), matrix $\mathbf{F}(\boldsymbol{\alpha})$ does not depend on the azimuth up to the order $P-3$ in $r_{1} / r$, and, from (49), $\theta$ and $(\phi, r)$ are decoupled up to the order $P-1$ in $r_{1} / r$. Consequently, the azimuth's CRB does not depend on the azimuth up to the order $P-1$ in $r_{1} / r$, in contrast to the elevation's and range's CRB for which this order is smaller or equal to $P-3$. Consequently, for fixed $r$ (resp. $P$ ), isotropy increases when $P$ (resp. $r$ ) increases. Also, azimuth's CRB is much less sensitive to the azimuth angle than elevation and range CRBs.

We introduce the following non-isotropy measurement, in which $\overline{\operatorname{CRB}(\theta)}$ denotes the mean of $\operatorname{CRB}(\theta)$ w.r.t. $\theta$

$$
\rho=\sup _{\theta} \frac{|\mathrm{CRB}(\theta)-\overline{\mathrm{CRB}(\theta)}|}{\overline{\mathrm{CRB}(\theta)}}
$$

illustrated in Fig.10 and Fig.11 for single-ring UCAs and uniform square based arrays with half-wavelength inter-sensors spacing, respectively. Fig.10 shows that the isotropy is much more sensitive to $P$ than to $r$, which increases very rapidly with $r$ and $P$ in contrast to Fig.11 where the isotropy is less sensitive to $Q$ and increases much less rapidly with $r$ and $Q$. In other words, the UCAs are much more isotropic than the uniform square-based arrays for given half aperture and range, under the near-field conditions.

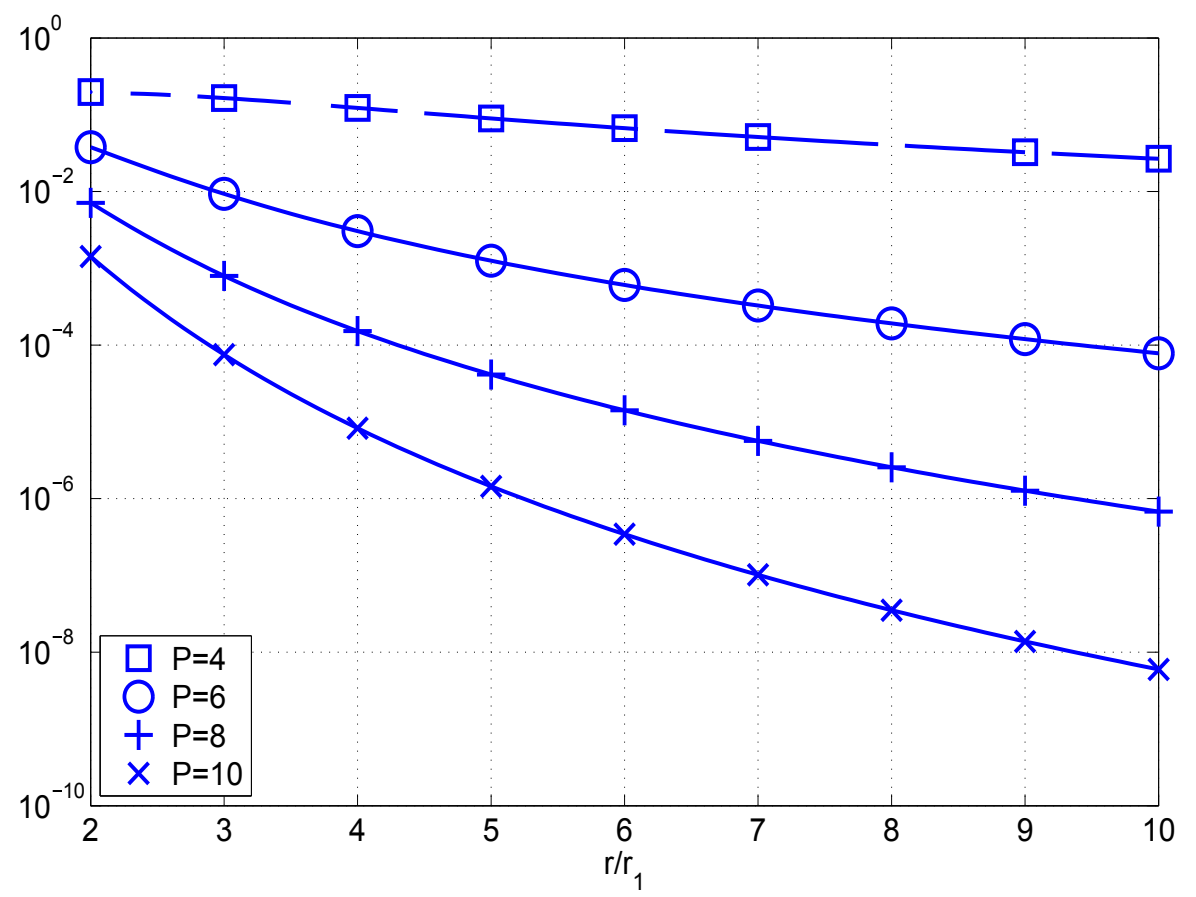

Fig.10 Non-isotropy criterion $\rho$ w.r.t. $r / r_{1}$ and $P$ for UCAs. 


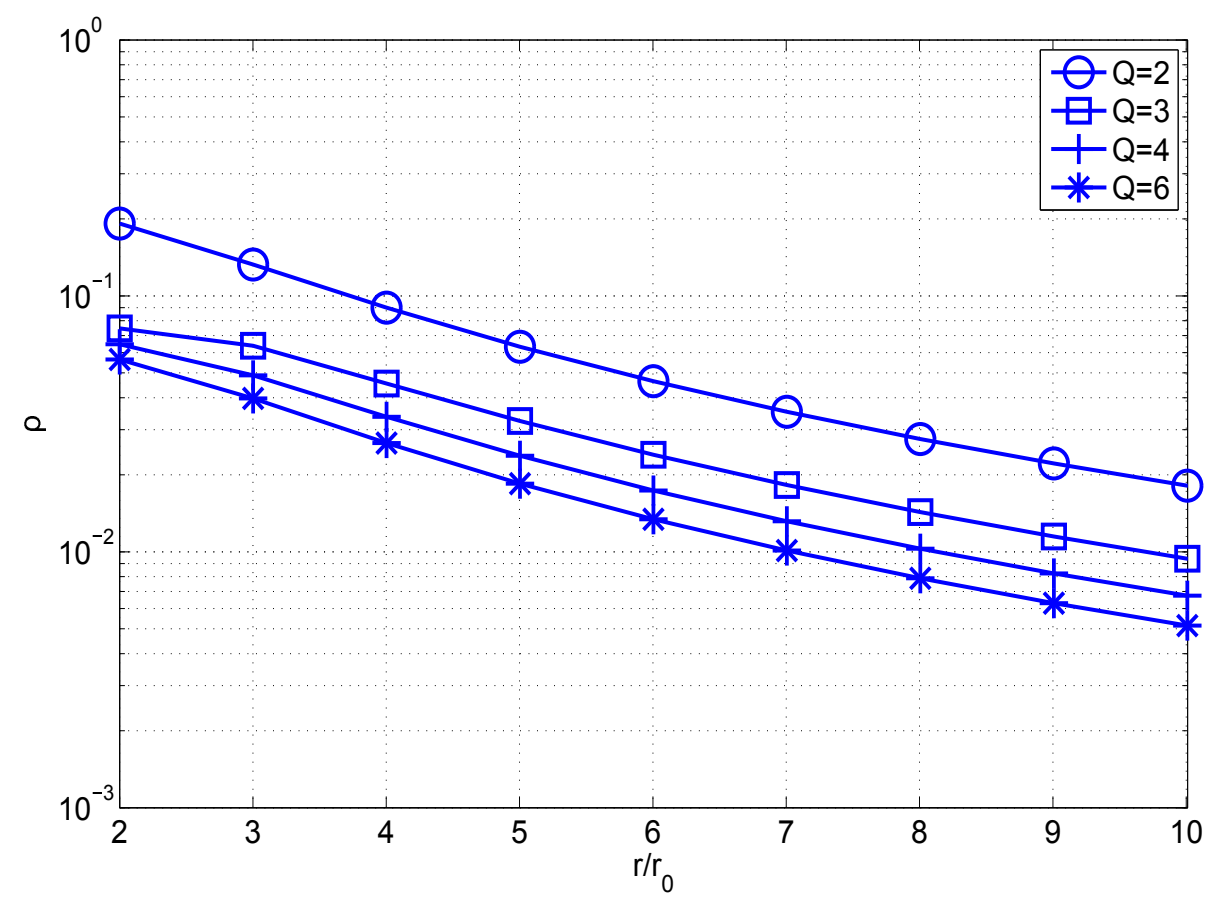

Fig.11 Non-isotropy criterion $\rho$ w.r.t. $r / r_{0}$ and $Q$ for uniform square-based arrays.

\section{B. Optimization of cross-based and square-based centro-symmetric arrays}

1) Optimization Criterion: Far-field (azimuth and elevation) performance is fully determined by the geometric parameter $\Sigma_{2}$ and the number $P$ of sensors as expressed in (45), while near-field performance depends on geometric parameters $\kappa$ and $\eta$ for DOA and range estimation. In particular, the most significant term of the range $\mathrm{CRB}$, as expressed in (44) and (46), is controlled by $\kappa$ through the term ${ }^{5}$

$$
\frac{r^{4}}{d(\theta, \phi, \kappa) \Sigma_{2}^{2}}=\frac{c r^{4}}{\Sigma_{2}^{2}}\left[\frac{1}{\kappa} a_{4}^{3,3}(\theta, \phi)+a_{2^{2}}^{3,3}(\theta, \phi)\right]^{-1},
$$

which is an increasing function of $\kappa$ as $a_{4}^{3,3}(\theta, \phi)>0$ for arbitrary $\theta$ and $\phi$.

Our array geometry optimization approach is inspired by the following rationale. For those arrays with predetermined values of $\Sigma_{2}$ and $P$ (and so, ones with similar far-field performance), near-field range estimation depends mainly on $\Sigma_{2}$ and $\kappa$. For comparison purposes, we refer to uniform cross (UCrA) and square-based (USA) arrays, for which $\kappa$ is denoted $\kappa_{u}$. We seek array geometries of nonuniform cross and square-based arrays, for which the $\kappa$-dependent criterion below is lower than one ${ }^{6}$.

$$
\mathcal{R}_{P}(\kappa)=\lim _{r \rightarrow \infty} \frac{\left.\operatorname{CRB}(r)\right|_{n u}}{\left.\operatorname{CRB}(r)\right|_{u}}=\frac{\frac{1}{\kappa_{u}} a_{4}^{3,3}(\theta, \phi)+a_{2^{2}}^{3,3}(\theta, \phi)}{\frac{1}{\kappa} a_{4}^{3,3}(\theta, \phi)+a_{2^{2}}^{3,3}(\theta, \phi)} .
$$

While spanning $\left[\frac{2}{Q}, 1\right]$, extreme values of $\kappa$ are to be avoided in order to preserve the DOA non-ambiguity of the cross-based and square-based centro-symmetric arrays. More specifically, on the one hand, $\kappa \approx \frac{2}{Q}$

${ }^{5}$ where $a_{4}^{3,3}(\theta, \phi)$ and $a_{2^{2}}^{3,3}(\theta, \phi)$ are defined in (31).

${ }^{6}$ where $\left.\operatorname{CRB}(r)\right|_{u}$ and $\left.\operatorname{CRB}(r)\right|_{n u}$ denote the $\mathrm{CRB}$ on $r$ for respectively uniform and nonuniform cross and square-based arrays. 
corresponds to co-located sensors at the centroid $O$, (except 4 sensors at $\left(x_{p}, y_{p}\right)=( \pm a, 0),(0, \pm a)$ for crossbased centro-symmetric arrays [resp., $\left(x_{p}, y_{p}\right)=( \pm a, \pm a)$ for square-based centro-symmetric arrays]). On the other hand, $\kappa \approx 1$ corresponds to $a_{q} \approx \pm a$ (all sensors concentrated to the previous four positions). Consequently, we only seek values of $\kappa$ in [0.3-0.7].

The ratio (52) illustrated in Fig.12, shows that there exist non-uniform square or cross-based centrosymmetric arrays with $\mathcal{R}_{P}(\kappa)$ significantly lower than 1 , suggesting that there is an opportunity to achieve a great deal of improvement. This is, actually true regardless of the source DOA as confirmed by Fig.13. This figure shows that $\mathcal{R}_{P}(\kappa)$ depends very loosely on $\theta$ and $\phi$. More precisely, it is not sensitive to $\theta$ due to the isotropy property, but little sensitive to $\phi$. Furthermore the performance advantage increases for weak values of $\phi$. The same behavior has been observed for cross-based centro-symmetric arrays.

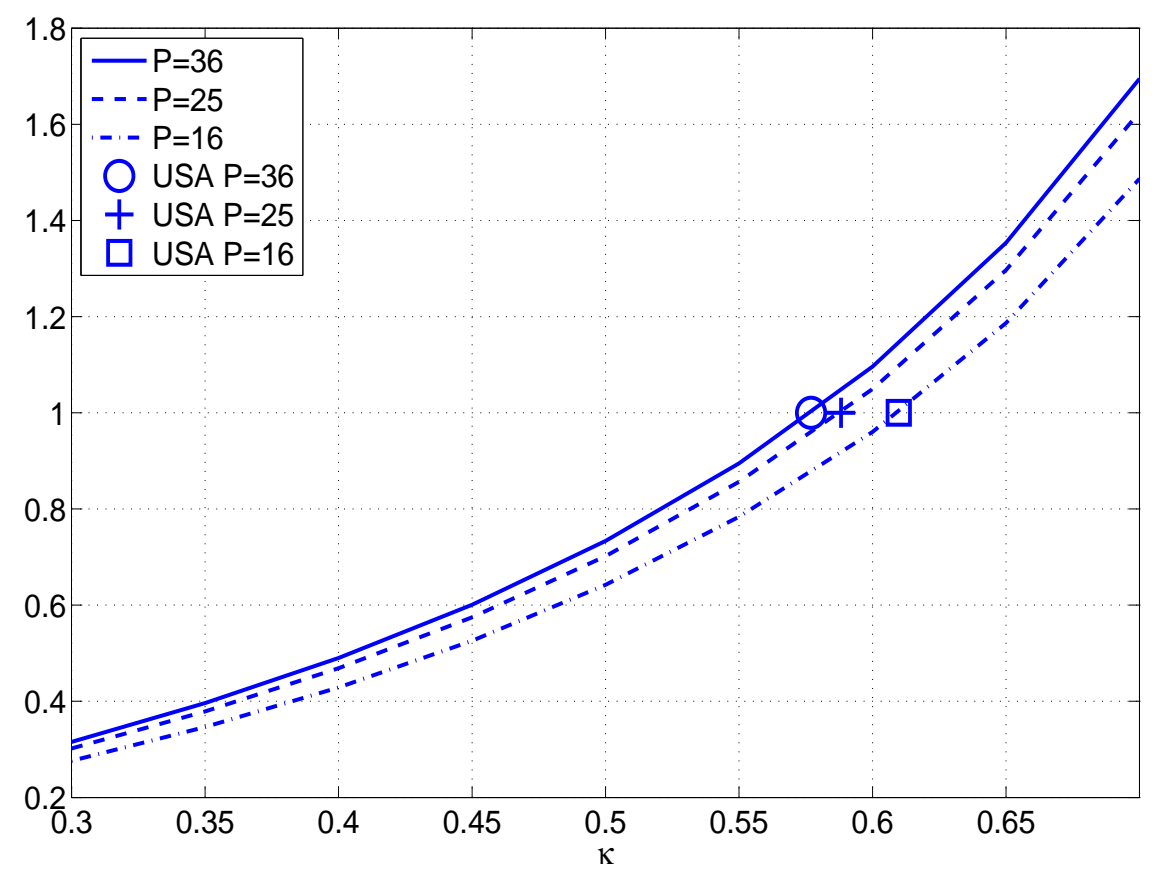

Fig.12 $\mathcal{R}_{P}(\kappa)$ as a function of $\kappa$ for different square-based centro-symmetric arrays for $\theta=60^{\circ}$ and $\phi=30^{\circ}$. 


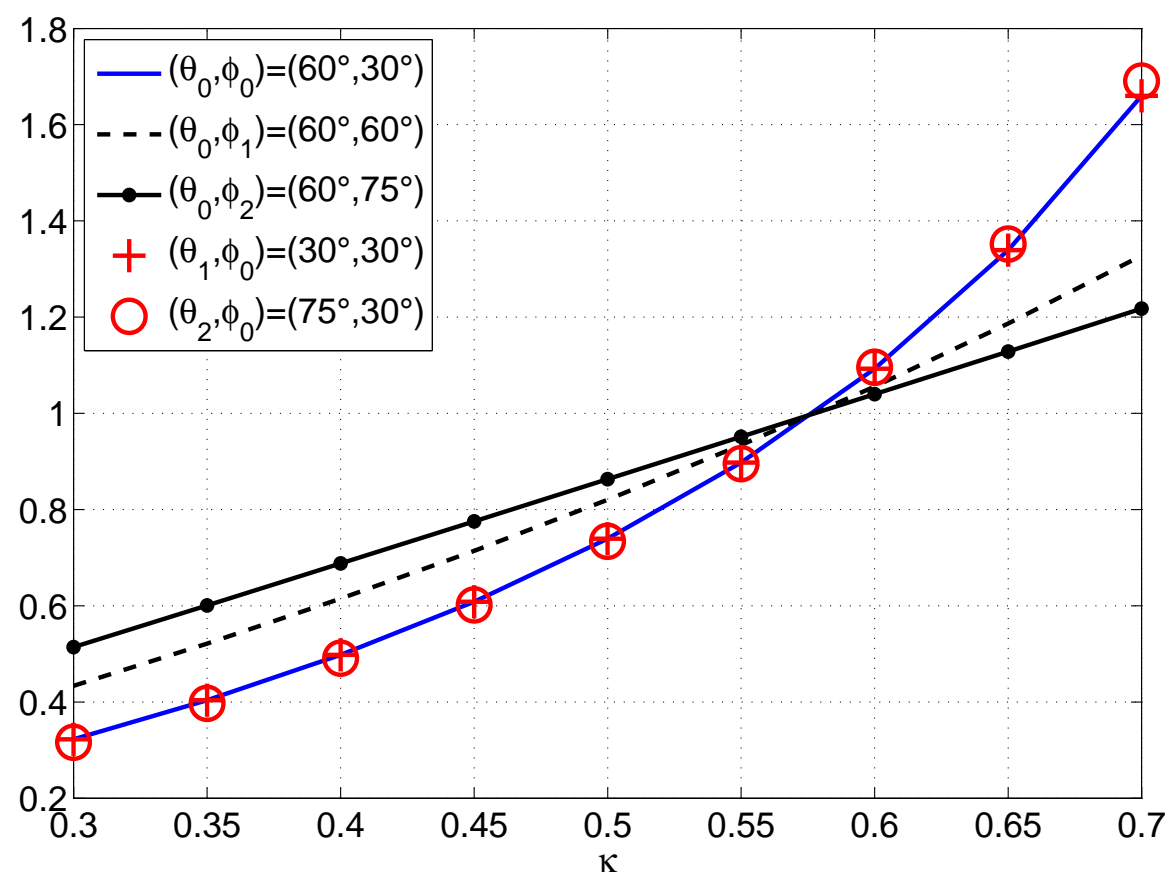

Fig.13 $\mathcal{R}_{P}\left(\kappa_{n u}\right)$ as a function of $\kappa_{n u}$ for square-based centro-symmetric arrays with $P=36$ for different $(\theta, \phi)$.

2) Sensors placement: Having fixed $\Sigma_{2}, P$ and $\kappa \in\left(\frac{2}{Q}, \kappa_{u}\right)$, based on desired near-field and far-field performance, there are $(Q / 2)-2$ for $Q$ even, [resp., $((Q-1) / 2)-2$ for $Q$ odd] degrees of freedom for arbitrary cross or square-based centro-symmetric arrays to set positions $a_{1}, \ldots a_{Q}$ of the sensors ${ }^{7}$. They are used to tackle the array ambiguity problem, a crucial one for the nonuniform array configurations.

Ambiguities occur when two steering vectors happen to be (very) close, despite referring to well separated look directions [18]. One way to minimize ambiguities is to minimize the so-called relative peak sidelobe level (PSL) ratio [5] derived from the conventional array beampattern [19]; If

$$
\left[\mathbf{a}_{\mathrm{FF}}(\theta, \phi)\right]_{p} \stackrel{\text { def }}{=} \lim _{r \rightarrow \infty}[\mathbf{a}(\boldsymbol{\alpha})]_{p}=e^{\frac{i 2 \pi}{\lambda}\left(\sin \phi\left(x_{p} \cos \theta+y_{p} \sin \theta\right)\right)},
$$

then

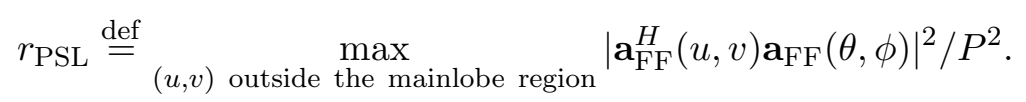

Since

$$
\min _{a_{1}, \ldots a_{Q}} r_{\mathrm{PSL}}
$$

under the constraints $\sum_{q=1}^{Q} a_{q}^{2}=\Sigma_{2}, \sum_{q=1}^{Q} a_{q}^{4}=\Sigma_{4}$ [with $\Sigma_{4}=\Sigma_{2}^{2} / Q \kappa$ from (41)] and symmetric $a_{q}$ is a nonconvex minimization problem ${ }^{8}$, we propose the following ad hoc criterion that ought to avoid concentrations

${ }^{7}$ except for $Q=4$ and 5, for which there remains no degree of freedom.

${ }^{8}$ including for $Q=6$ and 7, for which there is a single degree of freedom, but with several local minimum. 
of sensors in the neighborhood of the origin for weak values of $\kappa$ :

$$
\max _{a_{1}, \ldots a_{Q}}\left[\min _{1 \leq q \neq q^{\prime} \leq Q}\left|a_{q}-a_{q^{\prime}}\right|\right],
$$

under the same constraints. Results of the exhaustive search, reported in Tables 2 and 3, for $Q=6$ and 7, show that the proposed criterion (54) delivers very close values to those of the minimization (53).

To handle the max-min problem defined by (54) under the previous constraints, we introduce a new decision variable, denoted by $z$, in order to transform the aforementioned constrained optimization into a global polynomial maximization under, both, polynomial equalities and inequalities. This can be expressed as follows:

$$
\max z \text { under the following constraints }
$$

$z \leq 2 a_{1}, z \leq a_{q+1}-a_{q}, q=1, . ., Q / 2, \sum_{q=1}^{Q / 2} a_{q}^{2}=\frac{\Sigma_{2}}{2}, \sum_{q=1}^{Q / 2} a_{q}^{4}=\frac{\Sigma_{4}}{2}$ and centro-symmetric $a_{q}$ for $Q$ even $z \leq a_{1}, z \leq a_{q+1}-a_{q}, q=1, . .,\lfloor Q / 2\rfloor, \sum_{q=1}^{\lfloor Q / 2\rfloor} a_{q}^{2}=\frac{\Sigma_{2}}{2}, \sum_{q=1}^{\lfloor Q / 2\rfloor} a_{q}^{4}=\frac{\Sigma_{4}}{2}$ and centro-symmetric $a_{q}$ for $Q$ odd.

This is a constrained non-convex but polynomial optimization problem. Following [20], it can be solved by a sequence of semidefinite positive (SDP) relaxations. The result comes with global convergence guarantees and often at finite relaxation order. This method can be implemented using the matlab GloptiPoly utility [21]. By judiciously choosing the relaxation orders, we have solved our optimization problem with small relaxation order for $Q=6,7,8$ and 9 sensors.

Tables 2-5 assume a normalized $\Sigma_{2}=1$ and report for different values of $\kappa$ the associated $\mathcal{R}_{P}(\kappa)$, optimal sensors positions and the relative PSL for both cross-based (CrCS) and square-based (SCS) centro-symmetric arrays (denoted by $r_{\mathrm{PSL}}^{\mathrm{CrCS}}$ and $r_{\mathrm{PSL}}^{\mathrm{SCS}}$, respectively), for different values of the number $Q$ of sensors, $\theta=60^{\circ}$ and $\phi=30^{\circ}$.

\begin{tabular}{|c|c|c|c|c|c|}
\hline$\kappa$ & $\mathcal{R}_{P}(\kappa)$ & sensors positions & $r_{\mathrm{PSL}}^{\mathrm{CrCS}}$ & $r_{\mathrm{PSL}}^{\mathrm{SCS}}$ & criterion \\
\hline 0.5776 & 1 & $\pm 0.1195, \pm 0.3586, \pm 0.5976$ & 2.9087 & 17.3310 & $(54)$ \\
\hline 0.5000 & 0.7392 & $\pm 0.1012, \pm 0.3036, \pm 0.6305$ & 2.8441 & 6.6667 & $(54)$ \\
\hline 0.4500 & 0.6080 & $\pm 0.0866, \pm 0.2599, \pm 0.6519$ & 2.3719 & 4.1762 & $(54)$ \\
\hline 0.4000 & 0.4978 & $\pm 0.0674, \pm 0.2022, \pm 0.6742$ & 1.7310 & 3.7037 & $(54)$ \\
\hline 0.4000 & 0.4978 & $\pm 0.1335, \pm 0.1640, \pm 0.6748$ & 1.8115 & & $(53)$ \\
\hline 0.4000 & 0.4978 & $\pm 0.0823, \pm 0.1961, \pm 0.6744$ & & 3.8124 & $(53)$ \\
\hline 0.3500 & 0.4036 & $\pm 0.0349, \pm 0.1047, \pm 0.6984$ & 1.1689 & 1.3889 & $(54)$ \\
\hline 0.3500 & 0.4036 & $\pm 0.0332, \pm 0.1052, \pm 0.6984$ & 1.1698 & & $(53)$ \\
\hline 0.3500 & 0.4036 & $\pm 0.0701, \pm 0.0849, \pm 0.6984$ & & 1.3952 & $(53)$ \\
\hline
\end{tabular}

Table 2 Values of $\kappa, \mathcal{R}_{P}(\kappa)$, sensors positions, $r_{\mathrm{PSL}}^{\mathrm{CrCS}}$ and $r_{\mathrm{PSL}}^{\mathrm{SCS}}$ for $Q=6$. 


\begin{tabular}{|c|c|c|c|c|c|}
\hline$\kappa$ & $\mathcal{R}_{P}(\kappa)$ & sensors positions & $r_{\mathrm{PSL}}^{\mathrm{CrCS}}$ & $r_{\mathrm{PSL}}^{\mathrm{SCS}}$ & criterion \\
\hline 0.5714 & 1 & $0, \pm 0.1890, \pm 0.3780, \pm 0.5669$ & 3.3400 & 16.6945 & $(54)$ \\
\hline 0.5000 & 0.7571 & $0, \pm 0.1674, \pm 0.3348, \pm 0.5999$ & 2.6274 & 10.7527 & $(54)$ \\
\hline 0.4500 & 0.6228 & $0, \pm 0.1500, \pm 0.3001, \pm 0.6225$ & 2.3674 & 6.7340 & $(54)$ \\
\hline 0.4000 & 0.5098 & $0, \pm 0.1288, \pm 0.2577, \pm 0.6458$ & 2.5001 & 4.6751 & $(54)$ \\
\hline 0.4000 & 0.5098 & $0, \pm 0.1876, \pm 0.2150, \pm 0.6469$ & 2.7278 & & $(53)$ \\
\hline 0.4000 & 0.5098 & $0, \pm 0.1036, \pm 0.2705, \pm 0.6450$ & & 8.1699 & $(53)$ \\
\hline 0.3500 & 0.4133 & $0, \pm 0.1001, \pm 0.2003, \pm 0.6707$ & 1.7784 & 3.4868 & $(54)$ \\
\hline 0.3500 & 0.4133 & $0, \pm 0.1278, \pm 0.1828, \pm 0.6709$ & 1.8255 & & $(53)$ \\
\hline 0.3500 & 0.4133 & $0, \pm 0.1376, \pm 0.1753, \pm 0.6710$ & & 3.7298 & $(53)$ \\
\hline
\end{tabular}

Table 3 Values of $\kappa, \mathcal{R}_{P}(\kappa)$, sensors positions, $r_{\mathrm{PSL}}^{\mathrm{CrCS}}$ and $r_{\mathrm{PSL}}^{\mathrm{SCS}}$ for $Q=7$.

\begin{tabular}{|c|c|c|c|c|}
\hline$\kappa$ & $\mathcal{R}_{P}(\kappa)$ & sensors positions & $r_{\mathrm{PSL}}^{\mathrm{CrCS}}$ & $r_{\mathrm{PSL}}^{\mathrm{SCS}}$ \\
\hline 0.5676 & 1 & $\pm 0.0772, \pm 0.2315, \pm 0.3858, \pm 0.5401$ & 3.0202 & 18.1818 \\
\hline 0.5000 & 0.7685 & $\pm 0.0699, \pm 0.2098, \pm 0.3497, \pm 0.5734$ & 2.3964 & 12.5000 \\
\hline 0.4500 & 0.6325 & $\pm 0.0641, \pm 0.1922, \pm 0.3203, \pm 0.5969$ & 2.1739 & 7.1429 \\
\hline 0.4000 & 0.5176 & $\pm 0.0572, \pm 0.1715, \pm 0.2858, \pm 0.6210$ & 2.2727 & 6.2893 \\
\hline 0.3500 & 0.4197 & $\pm 0.0484, \pm 0.1452, \pm 0.2421, \pm 0.6465$ & 2.1186 & 5.8824 \\
\hline 0.3000 & 0.3352 & $\pm 0.0358, \pm 0.1074, \pm 0.1790, \pm 0.6747$ & 1.5362 & 2.5707 \\
\hline
\end{tabular}

Table 4 Values of $\kappa, \mathcal{R}_{P}(\kappa)$, sensors positions, $r_{\mathrm{PSL}}^{\mathrm{CrCS}}$ and $r_{\mathrm{PSL}}^{\mathrm{SCS}}$ for $Q=8$.

\begin{tabular}{|c|c|c|c|c|}
\hline$\kappa$ & $\mathcal{R}_{P}(\kappa)$ & sensors positions & $r_{\mathrm{PSL}}^{\mathrm{CrCS}}$ & $r_{\mathrm{PSL}}^{\mathrm{SCS}}$ \\
\hline 0.5650 & 1 & $0, \pm 0.1291, \pm 0.2582, \pm 0.3873, \pm 0.5164$ & 3.3344 & 19.4553 \\
\hline 0.5000 & 0.7767 & $0, \pm 0.1187, \pm 0.2374, \pm 0.3561, \pm 0.5502$ & 2.8571 & 10.9649 \\
\hline 0.4500 & 0.6387 & $0, \pm 0.1101, \pm 0.2202, \pm 0.3304, \pm 0.5747$ & 2.4190 & 9.4787 \\
\hline 0.4000 & 0.5228 & $0, \pm 0.1003, \pm 0.2006, \pm 0.3009, \pm 0.5993$ & 2.3759 & 8.5911 \\
\hline 0.3500 & 0.4240 & $0, \pm 0.0883, \pm 0.1766, \pm 0.2649, \pm 0.6252$ & 2.6247 & 6.2112 \\
\hline 0.3000 & 0.3386 & $0, \pm 0.0721, \pm 0.1442, \pm 0.2163, \pm 0.6536$ & 1.9231 & 4.4248 \\
\hline
\end{tabular}

Table 5 Values of $\kappa, \mathcal{R}_{P}(\kappa)$, sensors positions, $r_{\mathrm{PSL}}^{\mathrm{CrCS}}$ and $r_{\mathrm{PSL}}^{\mathrm{SCS}}$ for $Q=9$.

As seen in these tables, our objective of reducing the near-field range's CRB is achieved (to up to $60 \%$ ), while maintaining no-ambiguity of the cross-based and square-based centro-symmetric arrays. The reduction of the CRB increases with the number of sensors and robustness to ambiguity is much more better for square than for cross-based centro-symmetric arrays due to a larger number of sensors for a given $Q$. A tradeoff should be sought between performance improvement and the robustness to ambiguity.

We need to make sure that sensor positions that reduce the near-field range's CRB do not deteriorate the nearfield DOA's CRBs, comparatively to the UCrA or a USA. This is the case, as verified by extended numerical experiments, and illustrated in Fig. 14 exhibiting the three ratios $\left.\operatorname{CRB}(\theta)\right|_{n u} /\left.\operatorname{CRB}(\theta)\right|_{u},\left.\operatorname{CRB}(\phi)\right|_{n u} /\left.\operatorname{CRB}(\phi)\right|_{u}$ and $\left.\operatorname{CRB}(r)\right|_{n u} /\left.\operatorname{CRB}(r)\right|_{u}$ for the square-based centro-symmetric array with sensors of $P=Q^{2}=36$ sensors placed at $\pm 0.0674, \pm 0.2022$ and \pm 0.6742 with $\Sigma_{2}=1$, associated with $\kappa=0.4000$ for $\theta=60^{\circ}$ and $\phi=30^{\circ}$. This figure shows that the near-field range's CRB is reduced by a much as $50 \%$ without deteriorating the near-field DOA's CRB w.r.t. those of the USA. 


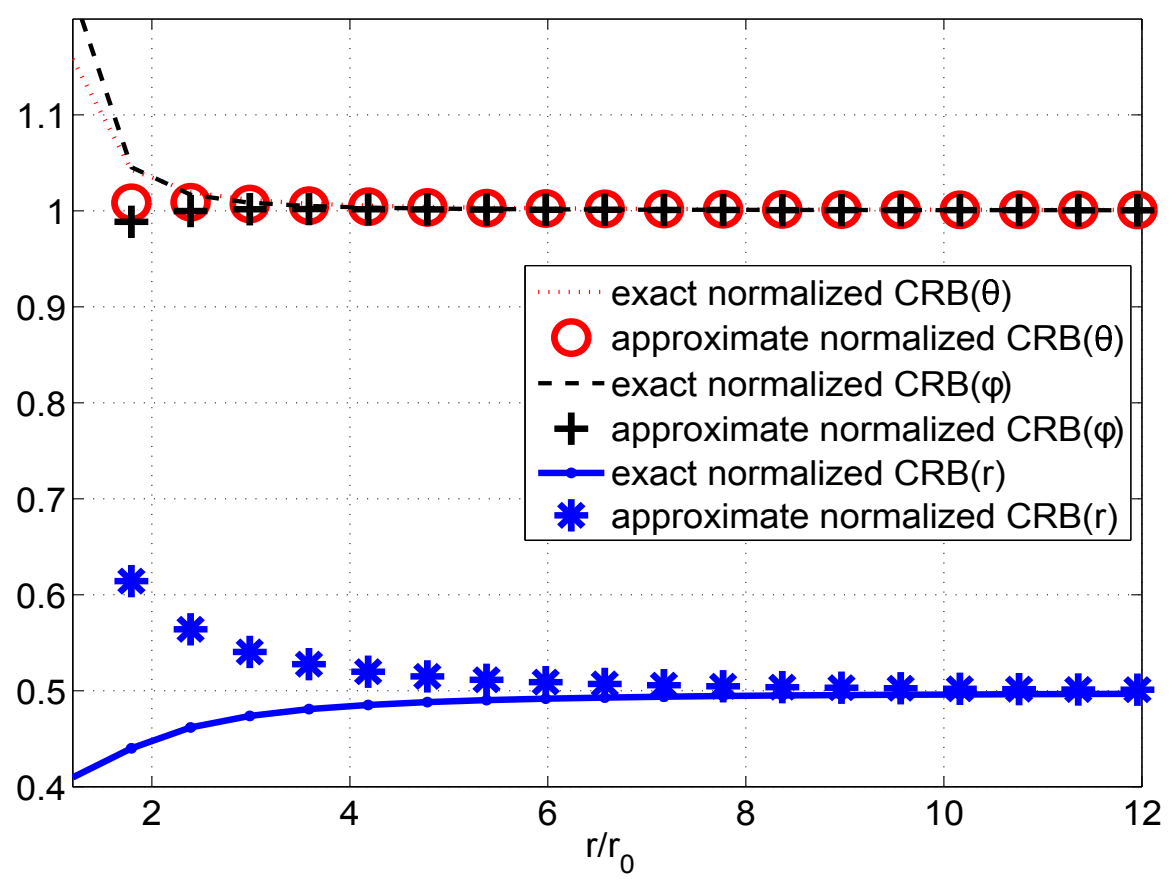

Fig.14 Azimuth, elevation and range CRBs of a square-based centro-symmetric arrays with $\kappa_{n u}=0.4000$ normalized to that of the equivalent USA $\left(\kappa_{u}=0.5776\right)$. Both arrays are made of $P=Q^{2}=36$ sensors

\section{CONCLUSION}

This paper has been dedicated to derivations and analysis of the azimuth, elevation and range CRBs for narrowband near-field source localization by means of planar arrays, where we have assumed the exact expression of the time delay parameter. Conditions on the array geometry that allow us to decouple the azimuth, elevation and range to a certain order in $1 / r$ have been highlighted, using Taylor expansions w.r.t. $1 / r$. These conditions complement those found for a near-field source that ensure the azimuth and elevation estimations are both exactly decoupled and isotropic. Explicit non-matrix closed-form expressions of these CRBs are derived for concentric uniform circular-based arrays, cross-based and square-based centro-symmetric arrays that satisfy these conditions. Using a new criterion that controls the direction of arrival (DOA) ambiguity, non-uniform square or cross-based centro-symmetric arrays are characterized with significantly lower range's CRB (by as much as 60\%) without deteriorating the DOA precisions w.r.t. uniform square or cross-based arrays. 


\section{APPENDIX}

\section{A. Taylor expressions of the terms of $\mathbf{F}(\boldsymbol{\alpha})$}

From (5) with $\tau_{p, 1}^{\prime}=2 \pi \frac{r}{\lambda} \frac{\sin \phi\left(-\frac{x_{p}}{r} \sin \theta+\frac{y_{p}}{r} \cos \theta\right)}{\sqrt{\beta_{p}}}, \tau_{p, 2}^{\prime}=2 \pi \frac{r}{\lambda} \frac{\cos \phi\left(\frac{x_{p}}{r} \cos \theta+\frac{y_{p}}{r} \sin \theta\right)}{\sqrt{\beta_{p}}}$ and $\tau_{p, 3}^{\prime}=$ $2 \pi \frac{1}{\lambda}\left(1+\frac{-1+\sin \phi\left(\frac{x_{p}}{r} \cos \theta+\frac{y_{p}}{r} \sin \theta\right)}{\sqrt{\beta_{p}}}\right)$, we obtain

$$
\begin{aligned}
{[\mathbf{F}]_{1,1}=} & \frac{r^{2}}{\lambda^{2}} 4 \pi^{2} c_{\sigma} \sin ^{2} \phi\left[P \sum_{p=1}^{P} \frac{\left(-\frac{x_{p}}{r} \sin \theta+\frac{y_{p}}{r} \cos \theta\right)^{2}}{\beta_{p}}-\left(\sum_{p=1}^{P} \frac{\left(-\frac{x_{p}}{r} \sin \theta+\frac{y_{p}}{r} \cos \theta\right)}{\sqrt{\beta_{p}}}\right)^{2}\right], \\
{[\mathbf{F}]_{1,3}=} & \frac{r}{\lambda^{2}} 4 \pi^{2} c_{\sigma} \sin \phi\left[P \sum_{p=1}^{P} \frac{\left(-\frac{x_{p}}{r} \sin \theta+\frac{y_{p}}{r} \cos \theta\right)}{\sqrt{\beta_{p}}}\left(1+\frac{-1+\sin \phi\left(\frac{x_{p}}{r} \cos \theta+\frac{y_{p}}{r} \sin \theta\right)}{\sqrt{\beta_{p}}}\right)\right. \\
& \left.-\sum_{p=1}^{P}\left(\frac{-\frac{x_{p}}{r} \sin \theta+\frac{y_{p}}{r} \cos \theta}{\sqrt{\beta_{p}}}\right)\left(\sum_{p=1}^{P} 1+\frac{-1+\sin \phi\left(\frac{x_{p}}{r} \cos \theta+\frac{y_{p}}{r} \sin \theta\right)}{\sqrt{\beta_{p}}}\right)\right], \\
{[\mathbf{F}]_{3,3}=\frac{1}{\lambda^{2}} 4 \pi^{2} c_{\sigma}\left[P \sum_{p=1}^{P}\left(1+\frac{-1+\sin \phi\left(\frac{x_{p}}{r} \cos \theta+\frac{y_{p}}{r} \sin \theta\right)}{\sqrt{\beta_{p}}}\right)^{2}\right.} & \left.-\left(\sum_{p=1}^{P} 1+\frac{-1+\sin \phi\left(\frac{x_{p}}{r} \cos \theta+\frac{y_{p}}{r} \sin \theta\right)}{\sqrt{\beta_{p}}}\right)^{2}\right] .
\end{aligned}
$$

Then we use the Taylor series expansions:

$$
1 / \beta_{p}=\sum_{k=0}^{+\infty}(-1)^{k} \gamma_{p}^{k} \text { and } 1 / \sqrt{\beta_{p}}=1+\sum_{k=1}^{+\infty} \frac{(-1)^{k} 1 \times 3 \times \ldots(2 k-1) \gamma_{p}^{k}}{2^{k} k !}
$$

where $\gamma_{p}=-2 \sin \phi\left(\frac{x_{p}}{r} \cos \theta+\frac{y_{p}}{r} \sin \theta\right)+\frac{x_{p}^{2}+y_{p}^{2}}{r^{2}}$ from the value of $\beta_{p}$ (2) in the expressions (56)-(57). This allows us to obtain Taylor series expansions of $[\mathbf{F}]_{1,1},[\mathbf{F}]_{1,3}$ and $[\mathbf{F}]_{3,3}$ w.r.t. $x_{p} / r$ and $y_{p} / r$. And thus, we can deduce the following structured Taylor series expansions: $[\mathbf{F}]_{1,1}=\frac{r^{2}}{\lambda^{2}} \sum_{k=1}^{\infty}\left[\frac{\sum_{\ell=0}^{k} g_{\ell, k}^{1,1}(\theta, \phi) S_{i, k-i}}{r^{k}}\right],[\mathbf{F}]_{1,3}=$ $\frac{r}{\lambda^{2}} \sum_{k=1}^{\infty}\left[\frac{\sum_{\ell=0}^{k} g_{\ell, k}^{1,3}(\theta, \phi) S_{i, k-i}}{r^{k}}\right]$ and $[\mathbf{F}]_{3,3}=\frac{1}{\lambda^{2}} \sum_{k=1}^{\infty}\left[\frac{\sum_{\ell=0}^{k} g_{\ell, k}^{3,3}(\theta, \phi) S_{i, k-i}}{r^{k}}\right]$, where $S_{i, j} \stackrel{\text { def }}{=} \sum_{p=1}^{P} x_{p}^{i} y_{p}^{j}$ are purely geometric parameters and $g_{\ell, k}^{i, j}(\theta, \phi)$ are trigonometric polynomial in $\theta$ and $\phi .[\mathbf{F}]_{1,2}$ and $[\mathbf{F}]_{2,2}$ are structured as $[\mathbf{F}]_{1,1}$ and $[\mathbf{F}]_{2,3}$ as $[\mathbf{F}]_{1,3}$.

\section{B. Concentric uniform circular-based arrays}

1) Proof of (20): From (5) and $\tau_{p, 1}^{\prime}=-2 \pi \frac{r_{i}}{\lambda} \frac{\sin \theta_{p, i} \sin \phi}{\sqrt{\beta_{p}}}$ for a sensor $C_{i}$ on the circle of radius $r_{i}$, we obtain

$$
\frac{1}{c_{\sigma}}[\mathbf{F}]_{1,1}=P \sum_{i=1}^{I}\left(\left(2 \pi \frac{r_{i}}{\lambda}\right)^{2} \sin ^{2} \phi \sum_{p \in C_{i}} \frac{\sin ^{2} \theta_{p, i}}{\beta_{p}}\right)-\left(\sum_{i=1}^{I}\left(2 \pi \frac{r_{i}}{\lambda} \sin \phi \sum_{p \in C_{i}} \frac{\sin \theta_{p, i}}{\sqrt{\beta_{p}}}\right)\right)^{2} .
$$

Taylor series expansion of $1 / \beta_{p}$ and $1 / \sqrt{\beta_{p}}$ w.r.t. $r_{i} / r$, where $\beta_{p}$ is given by (18), followed by elementary trigonometric relations, show that $[\mathbf{F}]_{1,1}$ depend on the azimuth $\theta$ only through the sums $\sum_{p_{i}=1}^{P_{i}} \cos k \theta_{p, i}$ and $\sum_{p_{i}=1}^{P_{i}} \sin k \theta_{p, i}$ for $k$ integer which can be easily simplified thanks to (19). This allows us to deduce (20) 
from (59) for $P_{i}>5$ after cumbersome but simple algebraic manipulations. The relations (21)-(25) are proved similarly.

2) Expressions of $g_{i}\left(\sin ^{2} \phi\right)$ polynomials: The polynomials $g_{i}\left(\sin ^{2} \phi\right), i=1,2,3$ and 4 are deduced from the Taylor expansion of $[\mathbf{F}]_{3,3}$ after simple but cumbersome derivations.

$$
\begin{aligned}
g_{1}\left(\sin ^{2} \phi\right) & =\frac{1}{4}-\frac{1}{4} \sin ^{2} \phi+\frac{3}{32} \sin ^{4} \phi \\
g_{2}\left(\sin ^{2} \phi\right) & =\left(\frac{1}{2}-\frac{1}{4} \sin ^{2} \phi\right)^{2} \\
g_{3, i}\left(\sin ^{2} \phi\right) & =-\frac{3}{8}+\frac{29}{16} \sin ^{2} \phi-\frac{147}{64} \sin ^{4} \phi+\frac{115}{128} \sin ^{6} \phi\left(1+\frac{1}{10} \mathbb{1}_{P_{i}=6} \cos 6 \theta\right) \\
g_{4}\left(\sin ^{2} \phi\right) & =\left(1-\frac{1}{2} \sin ^{2} \phi\right)\left(-\frac{3}{8}+\frac{9}{8} \sin ^{2} \phi-\frac{45}{64} \sin ^{4} \phi\right),
\end{aligned}
$$

where $\mathbb{1}_{P_{i}=6} \stackrel{\text { def }}{=} 1$ if $P_{i}=6$ and 0 otherwise.

\section{Cross-based and square-based centro-symmetric arrays}

Consider the term $[\mathbf{F}]_{1,1}$ given by (56). Using the expansions

$$
1 / \beta_{p}=1-\gamma_{p}+\gamma_{p}^{2}-\gamma_{p}^{3}+\gamma_{p}^{4}+o\left(\gamma_{p}^{4}\right) \text { and } 1 / \sqrt{\beta_{p}}=1-\frac{1}{2} \gamma_{p}+\frac{3}{8} \gamma_{p}^{2}+o\left(\gamma_{p}^{2}\right)
$$

with $\gamma_{p}=-2 \sin \phi\left(\frac{x_{p}}{r} \cos \theta+\frac{y_{p}}{r} \sin \theta\right)+\frac{x_{p}^{2}+y_{p}^{2}}{r^{2}}$ w.r.t. $\frac{x_{p}}{r}$ and $\frac{y_{p}}{r}$ in

$$
\sum_{p=1}^{P} \frac{\left(-\frac{x_{p}}{r} \sin \theta+\frac{y_{p}}{r} \cos \theta\right)^{2}}{\beta_{p}}=\sum_{p=1}^{P} \frac{x_{p}^{2}}{r^{2}} \frac{\sin ^{2} \theta}{\beta_{p}}+\sum_{p=1}^{P} \frac{y_{p}^{2}}{r^{2}} \frac{\cos ^{2} \theta}{\beta_{p}}-\sum_{p=1}^{P} \frac{x_{p} y_{p}}{r^{2}} \frac{\sin 2 \theta}{\beta_{p}}
$$

and

$$
\left(-\sum_{p=1}^{P} \frac{x_{p}}{r} \frac{\sin \theta}{\sqrt{\beta_{p}}}+\sum_{p=1}^{P} \frac{y_{p}}{r} \frac{\cos \theta}{\sqrt{\beta_{p}}}\right)^{2}
$$

we obtain after simple algebraic manipulations:

$\frac{c}{r^{2} \sin ^{2} \phi}[\mathbf{F}]_{1,1}=\frac{P S_{2}}{r^{2}}+\frac{P\left(S_{4}\left(2 \sin ^{2} \phi \sin ^{2} 2 \theta-1\right)+S_{2,2}\left(4 \sin ^{2} \phi\left(\sin ^{4} \theta+\cos ^{4} \theta-\sin ^{2} 2 \theta\right)-1\right)\right)}{r^{4}}+o\left(\epsilon^{4}\right)$,

where $S_{i} \stackrel{\text { def }}{=} S_{i, 0}=S_{0, i}$ for $i=2,4$ and $c \stackrel{\text { def }}{=} \frac{\lambda^{2}}{4 \pi^{2} c_{\sigma}}$. Consequently, we derive the common expression for the cross and square-based centro-symmetric arrays:

$$
\frac{c}{r^{2} \sin ^{2} \phi}[\mathbf{F}]_{1,1}=\frac{a_{2}^{1,1} \Sigma_{2}}{r^{2}}+\frac{a_{4}^{1,1}(\theta, \phi) Q \Sigma_{4}+a_{2^{2}}^{1,1}(\theta, \phi) \Sigma_{2}^{2}}{r^{4}}+o\left(\epsilon^{4}\right),
$$

where $a_{2}^{1,1}=P, a_{4}^{1,1}(\theta, \phi)=\frac{P}{Q}\left(2 \sin ^{2} \phi \sin ^{2} 2 \theta-1\right)$ and $a_{2^{2}}^{1,1}(\theta, \phi)=0$ for the cross-based centro-symmetric arrays, and $a_{2}^{1,1}=P Q, a_{4}^{1,1}(\theta, \phi)=P\left(2 \sin ^{2} \phi \sin ^{2} 2 \theta-1\right)$ and $a_{2^{2}}^{1,1}(\theta, \phi)=$ $P\left(4 \sin ^{2} \phi\left(\sin ^{4} \theta+\cos ^{4} \theta-\sin ^{2} 2 \theta\right)-1\right)$ for the square-based centro-symmetric arrays.

The other terms of the matrix $\mathbf{F}(\boldsymbol{\alpha})$ are derived similarly. We obtain:

$$
\frac{c}{r^{2} \cos ^{2} \phi}[\mathbf{F}]_{2,2}=\frac{P S_{2}}{r^{2}}+\frac{S_{4}\left(4 \sin ^{2} \phi\left(\sin ^{4} \theta+\cos ^{4} \theta\right)-1\right)+S_{2,2}\left(6 \sin ^{2} \phi \sin ^{2} 2 \theta-1\right)-S_{2}^{2} \sin ^{2} \phi}{r^{4}}+o\left(\epsilon^{4}\right)
$$




$$
\begin{gathered}
\frac{c}{r^{2} \sin ^{3} \phi \cos \phi}[\mathbf{F}]_{1,2}=P \frac{\left(3 S_{2,2}-S_{4}\right)}{r^{4}} \sin 4 \theta+o\left(\epsilon^{4}\right) \\
\frac{c}{r \sin ^{4} \phi}[\mathbf{F}]_{1,3}=-P \frac{3\left(3 S_{2,2}-S_{4}\right)}{8 r^{4}} \sin 4 \theta+o\left(\epsilon^{4}\right) \\
\frac{c}{r \sin \phi \cos \phi}[\mathbf{F}]_{2,3}=\frac{P\left(3 S_{2,2}\left(2-3 \sin ^{2} \phi \sin ^{2} 2 \theta\right)+8 S_{4}\left(1-\sin ^{2} \phi\left(\sin ^{4} \theta+\cos ^{4} \theta\right)\right)\right)-2 S_{2}^{2}\left(1+\cos ^{2} \phi\right)}{4 r^{4}} \\
\quad+o\left(\epsilon^{4}\right) \\
c[\mathbf{F}]_{3,3}=\frac{P\left(S_{4}\left(2+\sin ^{2} 2 \theta \cos 2 \phi+\cos ^{4} \phi\left(2-\sin ^{2} 2 \theta\right)\right)+S_{2,2}\left(3 \sin ^{2} 2 \theta\left(1+\cos ^{4} \phi\right)+2 \cos ^{2} \phi\left(3 \cos ^{2} 2 \theta-1\right)\right)\right)}{8 r^{4}} \\
-\frac{2 S_{2}^{2}\left(1+\cos ^{4} \phi+\cos 2 \phi\right)}{8 r^{4}} \\
+\frac{P S_{6}\left(-3+\frac{29}{4} \sin ^{2} 2 \theta+\cos ^{2} \phi\left(50+\cos ^{2} \phi\left(2 \cos ^{2} \phi-55\right)\right)+\frac{1}{4} \cos ^{2} \phi \sin ^{2} 2 \theta\left(-139+2 \cos ^{2} \phi\left(58-3 \cos ^{2} \phi\right)\right)\right)}{8 r^{6}} \\
+\frac{P S_{4,2}\left(20+\cos ^{2} \phi\left(11-49 \cos ^{2} \phi\right)-\frac{5}{4} \sin ^{2} 2 \theta\left(29+11 \cos ^{2} \phi-109 \cos ^{4} \phi+69 \cos ^{6} \phi\right)\right)}{8 r^{6}} \\
-\frac{3 S_{2} S_{4}\left(1+\cos ^{2} \phi\left(-3+\cos ^{2} \phi+5 \cos ^{4} \phi\right)+\frac{10}{4} \sin ^{2} 2 \theta \sin ^{2} \phi\left(\cos ^{4} \phi-1\right)\right)}{8 r^{6}} \\
-\frac{6 S_{2} S_{2,2}\left(-2+\frac{15}{4} \sin ^{2} 2 \theta\left(1+\cos ^{6} \phi-\cos ^{2} \phi\left(1+\cos ^{2} \phi\right)\right)+\cos ^{2} \phi\left(1+3 \cos ^{2} \phi\right)\right)}{8\left(\epsilon^{6}\right) .}
\end{gathered}
$$

These expressions allow us to prove the structured expressions (26)-(31).

\section{REFERENCES}

[1] X. Huang, J. Reilly, and M. Wong, "Optimal design of linear sensors," in International Conference on Acoustics, Speech and Signal Processing (ICASSP 1991), Toronto, Canada, May 1991.

[2] E. J. Vertatschitsch and S. Haykin, "Impact of linear array geometry on direction of arrival estimation for a single source," IEEE Trans. Antennas Propag., vol. 39, no. 5, pp. 576-584, May 1991.

[3] H. Gazzah and K. Abed-Meraim, "Optimum ambiguity free directional and omnidirectional planar antenna arrays for DOA estimation," IEEE Trans. Signal Process., vol. 57, no. 10, pp. 3942-3953, Oct. 2009.

[4] H. Gazzah and J.P. Delmas, "Direction finding antennas arrays for the randomly located sources," IEEE Trans. Signal Process., vol. 60, no. 11, pp. 6063-6068, Nov. 2012.

[5] X. Wang, E. Aboutanios, and M. G. Amin, ”Adaptive array thinning for enhanced DOA estimation," IEEE Signal Processing letters, vol. 22, no. 7, pp. 799-803, July 2014.

[6] E.L. Lehmann and G. Casella, Theory of Point Estimation, Springer Texts in Statistics, 2003.

[7] Y. Hua and T. Sarkar, "A note on the Cramer-Rao bound for 2-D direction finding based on 2-D array," IEEE Trans. Signal Process., vol. 39, no. 5, pp. 1215-1218, May 1991.

[8] H. Gazzah and S. Marcos, "Cramer-Rao bounds for antenna array design,” IEEE Trans. Signal Process., vol. 54, no. 1, pp. 336-345, Jan. 2006.

[9] E. Grosicki, K. Abed-Meraim, and Y. Hua, "A weighted linear prediction method for near-field source localization," IEEE Trans. Signal Process., vol. 53, no. 10, pp. 3651-3660, Oct. 2005.

[10] M. N. El Korso, R. Boyer, A. Renaux, and S. Marcos, "Conditional and unconditional Cramer Rao bounds for near-field Source localization," IEEE Trans. Signal Process., vol. 58, no. 5, pp. 2901-2906, May 2010.

[11] T. Bao, M. N. El Korso, and H. Ouslimani, "Cramer-Rao bound and statistical resolution limit investigation for near-field source localization", Digital Signal Processing, Elsevier, vol. 48, pp. 137-147, Jan. 2016.

[12] Y. Begriche, M. Thameri, and K. Abed-Meraim, "Exact conditional and unconditional Cramer Rao bound for near-field localization," Digital Signal Processing, no. 31, pp. 45-58, Aug. 2014.

[13] H. Gazzah and J.P. Delmas, "CRB-based design of linear antenna arrays for near-field source localization," IEEE Trans. Antennas Propag., vol. 62, no. 4, pp. 1965-1973, Apr. 2014.

[14] J.P. Delmas and H. Gazzah, "Analysis of near-field source localization using uniform circular arrays," International Conference on Acoustics, Speech and Signal Processing (ICASSP 2013), Vancouver, Canada, May 2013.

[15] H. Gazzah and J.P. Delmas, "Spectral efficiency of beamforming-based parameter estimation in the single source case," in Proc. IEEE SSP, Nice, pp. 153-156, 2011.

[16] B. Liao, K. M. Tsui and S. C. Chan, "Frequency invariant uniform concentric circular arrays with directional elements," IEEE Trans. Aerosp. Electron. Syst., vol. 69, no. 2, pp. 871-884, Apr. 2013. 
[17] A. Mirkin and L.H. Sibul, "Cramer-Rao bounds on angle estimation with a two-dimensional array," IEEE Trans. Signal Process., vol. 39, no. 2, pp. 515-517, Feb. 1991.

[18] M. Gavish and A. Weiss, "Array geometry for ambiguity resolution in direction finding," IEEE Trans. Antennas Propag., vol. 44, no. 6, pp. 889-895, June 1996.

[19] H. Messer, "Source localization performance and the beampattern," Signal Processing, vol. 28, no. 2, pp. 163-181, 1991.

[20] J.B. Lasserre, "Global optimization with polynomials and the problem of moments," SIAM Journal of Optimization vol. 11, no. 3, pp. 796-817, 2001.

[21] D. Henrion and J.B. Lasserre, "GloptiPoly: Global optimization over polynomials with Matlab and SeDuMi," ACM Transactions on Mathematical Software vol. 29, no. 2, pp. 165-194, June 2003. 